


\title{
QUASI DIMENSION TYPE. I. TYPES IN THE REAL LINE
}

\author{
JACK SEGAL
}

Fréchet defined the concept of dimension type in attempting to obtain a reasonable way of comparing two abstract topological spaces. The Fréchet dimension type of a topological space $X$ is said to be less than or equal to the Fréchet dimension type of the topological space $Y$ if and only if there is a homeomorphism between $X$ and a subset of $Y$. In this case we write $d X \leqq d Y$. Notice that the statement, $d X \leqq$ $d Y$, is equivalent to the statement, $X$ can be (topologically) embedded in $Y$.

Fréchet dimension type also is a more delicate way of comparing two spaces (when it applies) than covering dimension (denoted by dim). One difficulty is that many spaces are not comparable with respect to Fréchet dimension type because of the strong restriction of requiring that one be embeddable in the other. In this paper we will relax this restriction somewhat to obtain a new dimension type called quasi dimension type. Under quasi dimension type many more spaces are comparable and yet many of the properties of Fréchet dimension type are retained. Kuratowski showed that there are $2^{c}$ Fréchet dimension types represented by subsets of the real line. We will show that there are only denumerably many quasi dimension types represented by subsets of the real line. Furthermore, we completely determine the partial ordering of these types and give a topological characterization of the linear sets having a given type.

For two topological spaces $X$ and $Y$, we will say $X$ is quasi embeddable in $Y$, if, for each covering $\alpha$ of $X$, there is a closed $\alpha$ map, $f_{\alpha}$, of $X$ into $Y$. (Recall that a continuous function $f: X \rightarrow Y$ is an $\alpha$-map if there is a covering $\beta$ of $Y$ such that $f^{-1}[\beta[>\alpha$, i.e., $f^{-1}[\beta]$ refines $\alpha$, and $f$ is closed if it takes closed subsets of $X$ into closed subsets of $Y$.) We use "covering" to mean "open covering".

Furthermore, we will say that the quasi dimension type of a topological space $X$ is less than or equal to the quasi dimension type of a topological space $Y$ if and only if $X$ is quasi embeddable in $Y$. If this case we write $q X \leqq q Y$. We say $q X<q Y$ if $q X \leqq q Y$ but it is false that $q Y \leqq q X$. Further if $q X \leqq q Y$ and $q Y \leqq q X$ then $q X=$ $q Y$. So for two spaces $X$ and $Y$, one and only one of the following conditions holds: (1) $q X<q Y$, (2) $q Y<q X$, (3) $q X=q Y$ and (4) $X$ and $Y$ are not comparable with respect to quasi dimension type. Clearly, if $q X \leqq q Y$ and $q Y \leqq q Z$ then $q X \leqq q Z$. Note also that 
quasi dimension type is a topological invariant of $Y$ and is monotone on closed subsets, (i.e., if $X$ is a closed subset of $Y$ then $q X \leqq q Y$ ).

1. Preliminaries. All spaces considered in the remainder of this paper are metric. The diameter of $A$ will be denoted by $\delta(A)$.

DeFinition 1.1. A mapping $f: X \rightarrow Y$ is a strong $\varepsilon-m a p$ if there is an $\eta>0$ such that: if $A \subset Y$ and $\delta(A)<\eta$, then $\delta\left(f^{-1}[A]\right)<\varepsilon$. A mapping $f: X \rightarrow Y$ is an $\varepsilon-m a p$ provided that, for each $y \in Y$, we have $\delta\left(f^{-1}(y)\right)<\varepsilon$.

Definition 1.2. A space $X$ is strongly e-embeddable in a space $Y$ provided that, for each $\varepsilon>0$, there is a closed strong $\varepsilon$-map of $X$ into $Y$. If this is the case we write $s X \leqq s Y$.

REMARK. Note that $X$ and $Y$ may be homeomorphic without $s X \leqq s Y$ being true (see Example 1.1). In general, neither quasi embeddability nor strong $\varepsilon$-embeddability implies the other (see Examples 1.1 and 1.2). However, we do have (1) if $Y$ is compact, then $q X \leqq q Y$ implies $s X \leqq s Y$ and (2) if $X$ is compact, then $s X \leqq s Y$ implies $q X \leqq q Y$. If $X$ and $Y$ are compact then the following statements are equivalent: (3) $q X \leqq q Y$, (4) $s X \leqq s Y$ and (5) there are $\varepsilon$-maps of $X$ into $Y$ for all $\varepsilon>0$. If $q X \leqq q Y$, then (6) if for each $\alpha$, and for each $\alpha$-map of $X$ into $Y, f_{\alpha}$, we have that $f_{\alpha}[X]$ is compact (connected), then $X$ is compact (connected) and (7) $\operatorname{dim} X \leqq \operatorname{dim} Y$ (see [8] and [9] for more details on these mappings). It should be pointed out that in contrast to (7) that the existence of $\varepsilon$-maps of $X$ into $Y$ for all $\varepsilon>0$ does not, in general, imply that $\operatorname{dim} X \leqq \operatorname{dim} Y$ (see [12]).

Definition AND Notation 1.3. If $\alpha=\left\{A_{b}\right\}$ is a collection of sets and $X$ is a set, then $\alpha \cap X=\left\{A_{b} \cap X \mid A_{b} \in \alpha, A_{b} \cap X \neq \varnothing\right\}$. A set $A$ is countable if and only if card $A \leqq \boldsymbol{\aleph}_{0}$. A set $A$ is denumerable if and only if card $A=\boldsymbol{\aleph}_{0}$. A set is dense in itself if each of its points is a limit point of it. We define the sets (where $R^{1}$ denotes the real (Euclidean) line):

$$
\begin{aligned}
Q & =\left\{x \in R^{1} \mid x \text { is rational }\right\}, \\
J_{0} & =\varnothing=\text { empty set } \\
J & =\left\{x \in R^{1} \mid x \text { is a positive integer }\right\}, \\
J_{n} & =\{x \in J \mid 1 \leqq x \leqq n\}, \\
C & =\text { Cantor discontinuum on }[0,1], \\
D & =\left\{x \in R^{1} \mid x=0 \text { or } 1 / n \text { for } n=1,2, \cdots\right\}, \\
I & =[-1 / 2,0],
\end{aligned}
$$




$$
\begin{aligned}
J^{*} & =\bigcup\{[i, i+1 / 2] \mid i \in J\}, \\
L^{*} & =\bigcup\{[1 /(n+1), 1 / n) \mid n=1,3,5, \cdots\} \bigcup\{0\}, \\
M^{*} & =\bigcup\{(1 /(n+1), 1 / n) \mid n=1,3,5, \cdots\} \bigcup\{0\}, \\
L_{n} & =\bigcup\left\{(-2 j-1 / 2,-2 j] \mid j \in J_{n}\right\}, \\
L_{\infty} & =\bigcup\{(-2 j-1 / 2,-2 j] \mid j \in J\}, L_{0}=\varnothing \\
M_{n} & =\bigcup\left\{(-2 j+1 / 2,-2 j+1) \mid j \in J_{n}\right\}, \\
M_{\infty} & =\bigcup\{(-2 j+1 / 2,-2 j+1) \mid j \in J\}, M_{0}=\varnothing
\end{aligned}
$$

EXAmPle 1.1. $(q X \leqq q Y$ does not imply $s X \leqq s Y$.) Let $X=J$, $Y=D-\{0\}$ and $\alpha$ be any cover of $X$. Then $\alpha^{*}=\{\{i\} \mid i \in J\}$ refines $\alpha$ and $f: X \rightarrow Y$ defined by $f(i)=1 / i$ is a closed map. Let $\beta=$ $\{\{1 / i\} \mid i \in J\}$. Then $f^{-1}[\beta]>\alpha^{*}>\alpha$ and so $f$ is an $\alpha$-map. Thus we have $q X \leqq q Y$. Now we will assume $s X \leqq s Y$ and show it leads to a contradiction. Let $g$ be a closed (1/2)-map of $X$ and $Y$. Then, for any $\eta>0$, there are distinct positive integers $i$ and $j$ such that $|(1 / i)-(1 / j)|<\eta$ and $1 / i, 1 / j \in g[X]$. But $\delta\left(g^{-1}[\{1 / i\} \cup\{1 / j\}]\right) \geqq 1>1 / 2$. Thus $s X \leqq s Y$ must be false.

EXAmPle 1.2. $(s X \leqq s Y$ does not imply $q X \leqq q Y)$. Let $X=$ $D-\{0\}$ and $Y=D$. Then, for any $\varepsilon>0$, there is a closed strong $\varepsilon$-map, $f_{\varepsilon}: X \rightarrow Y$, defined by

$$
f_{\varepsilon}(1 / i)=\left\{\begin{array}{l}
0,0<1 / i<\varepsilon / 2 \\
1 / i, \varepsilon / 2 \leqq 1 / i \leqq 1
\end{array}\right\} .
$$

Let $\eta=\varepsilon / 2$ and suppose $A \subset Y$ and $\delta(A)<\eta$. Then if $0 \in A$ we have $A \subset[0, \eta)$ and so $\delta\left(f^{-1}[A]\right)<\varepsilon$. If $0 \notin A$ we have $\delta\left(f^{-1}[A]\right)<\eta<\varepsilon$. Hence $s X \leqq s Y$. Now we will assume that $q X \leqq q Y$ and show it leads to a contradiction. The image under each closed $\alpha$-map of $X$ is closed in $Y$ and hence compact. This implies that $X$ is compact which is a contradiction.

EXAMPLE 1.3. It is possible for $X$ to admit closed $\varepsilon$-mappings into $Y$, for all $\varepsilon>0$, and yet not admit $\varepsilon$-mappings onto a fixed subset of $Y$ for all $\varepsilon>0$. For example, let $X=D$ and $Y=J$. Just defined $f_{n}$ by

$$
f_{n}(x)=\left\{\begin{array}{l}
1, \text { for } 0 \leqq x<1 / n \text { and } x \in X \\
1+(1 / x), \text { for } 1 / n \leqq x \leqq 1 \text { and } x \in X
\end{array}\right\}
$$

Then $f_{n}$ is a $(1 / n)$-mapping of $X$ onto $J_{n+1} \subset Y$, (i.e., $\left.q D \leqq q J\right)$. Suppose that there is a subset $Y_{0}$ of $Y$ such that $X$ admits $\varepsilon$-mappings onto $Y_{0}$ for all $\varepsilon>0$. Since $X$ is compact so is $Y_{0}$. But the only 
compact subsets of $Y$ are finite. Obviously $X$ cannot be $\varepsilon$-mapped onto a finite set for all $\varepsilon>0$.

Example 1.4. Now while two spaces of the same Fréchet dimension type have the same cardinality, this may not be the case for two spaces of the same quasi dimension type. For example, consider the spaces $C$ and $D$. It is clear that $q D \leqq q C$ and we wish to show that $q C \leqq q D$. Recall that $C$ is obtained by first dividing $[0,1]$ into three equal subintervals and deleting the interior of the middle one. Then each of the two remaining intervals is divided into three equal intervals and the interiors of the middle ones are deleted. Then each of the remaining four intervals is treated similarly and so on indefinitely. The set of points remaining after this process is $C$. Now consider the $2^{n}$ disjoint closed intervals, $\left\{K_{i} \mid i=1, \cdots, 2^{n}\right\}$, remaining after the $n$th step in this construction of $C$. Define $f_{n}: C \rightarrow D$ by $f_{n}\left[K_{i} \cap C\right]=1 / i, i=1, \cdots, 2^{n}$. Then $f_{n}$ is an $\left(1 / 2^{n}\right)$-mapping since

$$
f_{n}^{-1}(1 / i)=\left\{\begin{array}{l}
K_{i}, i=1, \cdots, 2^{n} \\
\varnothing, i>2^{n}
\end{array}\right\}
$$

and $\delta\left(K_{i}\right)=1 / 3^{n}<1 / 2^{n}$. Thus we have that $q C \leqq q D$ since $C$ and $D$ are compact. Hence we may conclude that $q C=q D$.

Throughout this paper a compact connected space of more than one point will be called a continuum. A chain is a finite collection of open sets $U_{1}, \cdots, U_{n}$ such that $U_{i}$ intersects $U_{j}$ if and only if $i=j-1, j$, or $j+1$. If the links of a chain are of diameter less than $\varepsilon$, the chain is called an $\varepsilon$-chain. A continuum is called snakelike if for each $\varepsilon>0$ it can be covered by an $\varepsilon$-chain (see [1]).

EXAMPLE 1.5. Let $X$ be a snake-like continuum and $Y$ an arc. Then $X$ admits $\varepsilon$-mappings onto $Y$ for all $\varepsilon>0$ (see [5, p. 229] or [2, Lemma 1.6]). So $q X \leqq q Y$. If we specialize $X$ further to be a snake-like continuum which does not contain an arc then $q Y \leqq q X$ is false. So in this we have $q X<q Y$. However, $X$ and $Y$ are not comparable with respect to Fréchet dimension type.

We now give some general results which will be of use in the next section.

Theorem 1.1. If $q X \leqq q Y$ and $Y$ is compact (totally disconnected), then $X$ is compact (totally disconnected).

CoRollary 1.1. If $q X \leqq q Y$ and each component of $Y$ is compact, then each component of $X$ is compact. 
THEOREM 1.2. If (1) $X$ is the union of two disjoint closed sets $X_{1}$ and $X_{2}$, (2) $Y$ is the union of two disjoint closed sets $Y_{1}$ and $Y_{2}$, and (3) $q X_{i} \leqq q Y_{i}$, for $i=1,2$, then $q X \leqq q Y$.

Proof. Let $\alpha$ be a covering of $X$. Then $\alpha_{1}=\alpha \cap X_{1}$ and $\alpha_{2}=$ $\alpha \cap X_{2}$ are coverings of $X_{1}$ and $X_{2}$, respectively. Let $f_{i}$ be a closed $\alpha_{i}$-mapping of $X_{i}$ into $Y_{i}$ for $i=1,2$. Then, for $i=1,2$, there is a covering $\beta_{i}$ of $Y_{i}$ such that $f_{i}^{-1}\left[\beta_{i}\right]>\alpha_{i}$ and since $Y_{i}$ is open in $Y$, so is each element of $\beta_{i}$. Therefore, $\beta=\beta_{1} \cup \beta_{2}$ is a covering of $Y$ and the mapping which is $f_{1}$ on $X_{1}$ and $f_{2}$ on $X_{2}$ is a closed $\alpha$-mapping of $X$ into $Y$.

REMARK. Theorem 1.2 is not true with " $\leqq$ " replaced everywhere by " $<$ ". To see this let $X_{1}=I, X_{2}=J, Y_{1}=I \cup J$ and $Y_{2}=$ $\{x+3 / 4 \mid x \in(I \cup J)\}$. Then $q X_{i}<q Y_{i}, i=1,2$, but $q X=q Y$.

2. Quasi dimension type in the real line. We show in this section that there are only denumerably many quasi dimension types represented by subsets of $R^{1}$. This is in contradistinction to Kuratowski's result [6] that there are $2^{c}$ Frechet dimension types represented by subsets of $R^{1}$. Furthermore, we obtain a representative of each type, determine completely the ordering of these types and give a topological characterization of the linear sets having a given type. For the remainder of the paper all sets considered are subsets of $R^{1}$.

THEOREm 2.1. [10] If $A$ and $B$ are denumerable sets, each dense in itself, then $A$ and $B$ are homeomorphic.

Corollary 2.1. If $X$ is a countable closed set then $q X \leqq q Q$.

Proof. The set $X \cup Q$ is dense in itself and denumerable so $q(X \cup Q)=q Q$. Since $X$ is a closed set it is also a closed subset of $X \cup Q$. Thus we have $q X \leqq q(X \cup Q)=q Q$.

REMARK. So $q J_{i}, q D$ and $q J$ are all $\leqq q Q$. Using previous examples we have

$$
q J_{i}<q D=q C<q J=q(D-\{0\}) \leqq q Q .
$$

Theorem 2.2. $q X \leqq q J$ if and only if $X$ is totally disconnected.

Proof. We first assume that $X$ is totally disconnected. If $\alpha$ is a covering of $X$, then, since $\operatorname{dim} X \leqq 0$, there is a covering, $\alpha^{\prime}$, of $X$ 
which refines $\alpha$ and is such that no two elements $\alpha^{\prime}$ meet. Then by Lindelöf's theorem there is a countable subcollection $\alpha^{\prime \prime}$ of $\alpha^{\prime}$ which covers $X$. Let $f: X \rightarrow J$ be defined by $f\left[A_{i}\right]=i$ for $A_{i} \in \alpha^{\prime \prime}$. Then $f$ is a closed $\alpha$-map since any subset of $J$ is closed and $\beta=\{\{i\} \mid i \in J\}$ is such that $f^{-1}[\beta]>\alpha^{\prime \prime}>\alpha^{\prime}>\alpha$. Thus we have $q X \leqq q J$. The converse follows from Theorem 1.1.

THEOREM 2.3. $q X \leqq q C$ if and only if $X$ is compact and totally disconnected. A space $X$ is infinite (finite), compact and totally disconnected if and only if $q X=q C\left(q J_{i}\right.$, for some nonnegative integer i).

Proof. Any compact, totally disconnected, metric space $X$ is homeomorphic to a closed subset of $C$. So $q X \leqq q C$. If $X$ is infinite then it contains a copy of $D$. So $q D \leqq q X$ and it follows that $q X=$ $q C$. The converses are obvious from Theorem 1.1.

CoRollary 2.2. $q X=q J$ if and only if $X$ is totally disconnected and not compact.

Proof. Suppose $X$ is totally disconnected and not compact. By Theorem 2.2 we have $q X \leqq q J$. If $\alpha$ is any covering of $J$, then $\alpha^{\prime}=\{\{i\} \mid i \in J\}$ refines it. There is a sequence of points $x_{1}, x_{2}, \cdots$ of $X$ such that no subsequence converges to a point of $X$. Define $f: J \rightarrow K$ by $f(i)=x_{i}$. Then $f$ is a closed map since each subset of $\left\{x_{i} \mid i \in J\right\}$ is closed in $X$. There is a covering $\beta$ of $X$, each set of which contains at most one point $x_{i}$. Then $f^{-1}[\beta]>\alpha^{\prime}>\alpha$ and so $q J \leqq q X$. Thus we have $q J=q X$. The converse follows immediately from the two previous theorems.

We state as a separate corollary some of the information embodied in the proof of Corollary 2.2.

Corollary 2.3. If $X$ is not compact, then $X$ has a closed subset $Z$ such that $q J \leqq q Z$.

DeFinition 2.1. We say $q A$ immediately preceeds $q B$, written $q A \ll q B$, if $q A<q B$ and there is no set $X$ such that $q A<q X<q B$.

REMARK. For totally disconnected sets we have the following ordering:

$$
q J_{0} \ll q J_{1} \ll q J_{2} \ll \cdots \ll q J_{i} \ll q J_{i+1} \ll \cdots<q C \ll q J .
$$

Note that $q C$ is the least upper bound of the $q J_{i}$ 's. This follows from 
Theorem 2.3. That $q C \ll q J$ follows from Theorems 2.2 and 2.3 and Corollary 2.2. Moreover, if $X$ is a compact (noncompact) infinite totally disconnected set then $q X=q C(q J)$. Thus there are only denumerably many quasi dimension types of totally disconnected subsets of $R^{1}$.

THEOREM 2.4. $q X=q I$ if and only if $X$ is compact and not totally disconnected.

Proof. Assume $X$ is compact and not totally disconnected. Since $X$ contains a nondegenerate component, $K$, which is closed (in $X$ ) and hence compact we have $K=[a, b]$. So $q I=q[a, b] \leqq q X$. Now $X$ being compact is homeomorphic to a closed subset of $I$, so $q X \leqq q I$. The converse follows from Theorem 1.1.

THEOREM 2.5. $q J \leqq q X$ if and only if $X$ contains a closed subset homeomorphic to $J$.

Proof. One implication follows from the definition of quasi dimension type. To show the converse we proceed as in the proof of Theorem 2.3. Let $\alpha=\{\{i\} \mid i \in J\}$ denote the finest covering of $J$. Then there is a closed $\alpha$-map $f: J \rightarrow X$. Since $f$ is closed, one-to-one and continuous it is a homeomorphism.

Theorem 2.6. The space $J$ and any compact, not totally disconnected space $X$ are not comparable. In particular, $J$ and $I$ are not comparable.

Proof. Theorem 1.1 implies $q X \not \equiv \mathcal{J}$ (since $X$ is not totally disconnected) and $q J \geqq \equiv X$ (since $J$ is not compact).

THEOREM 2.7. $q C \ll q I$.

Proof. Since $C$ is homeomorphic to a closed subset of $I$ and $q I \geqq q C$ we have $q C<q I$. Suppose there is a set $X$ such that $q C<q X<q I$. Then by Theorem 1.1 we have that $X$ is compact. Case 1: $X$ is not totally disconnected; then $q X=q I$ which is a contradiction. Case 2: $X$ is totally disconnected. Then $q X \leqq q C$ which again is a contradiction. Thus we may conclude that $q C \ll q I$.

Theorem 2.8. $q J \ll q(I \cup J)$.

Proof. Since $J$ is a closed subset of $I \cup J$ and $q(I \cup J) \not \equiv J$ we have $q J<q(I \cup J)$. Suppose that there is a set $X$ such that 
$q J<q X<q(I \cup J)$. Then $X$ is not compact. Case 1: $X$ is not totally disconnected. Then $X$ contains nondegenerate arc components. If one of these, $K$, is not compact, then $q(I \cup J) \leqq q K \leqq q X$ (this last inequality is due to the fact that $K$ is closed in $X$ ). This is a contradiction. If, on the otherhand, $K$ is compact, then we take a closed, totally disconnected, noncompact subset $Z$ of $X-K$ (which is possible because $X$ is not compact) and have $q(I \cup J) \leqq q(K \cup Z) \leqq q X$. Again this is a contradiction. Case 2: $X$ is totally disconnected. Then $q X=q J$ which is a contradiction.

\section{THEOREM 2.9. $q I \ll q(I \cup J)$.}

Proof. Since $I$ is closed in $I \cup J$ and $q(I \cup J) \$ q I$ we have $q I<q(I \cup J)$. Suppose that there is a set $X$ such that $q I<q X<q(I \cup J)$. Then $X$ must be noncompact. If $X$ is totally disconnected, then $q X=q J$ which is impossible. Hence $X$ contains nondegenerate arc components. If one of these $(K)$ is not compact, $K$ has a closed subset homeomorphic to $I \cup J$, so $q(I \cup J) \leqq q K \leqq q X$. This is impossible. If, on the other hand, $K$ is compact, then we get a contradiction as before.

Notation. Let $Y$ be a subset of $X$. We use $N(Y)$ to denote the closure (in $X$ ) of the union of the nondegenerate components of $Y$.

\section{Lemma 2.1. If $q X \leqq q(I \cup J)$, then $q N(X) \leqq q I$.}

Proof. If $N(X)$ is compact we are done by Theorem 2.4. Suppose $N(X)$ is not compact. Then, since each component of $N(X)$ is compact (Corollary 1.1), there is a sequence $K_{1}, K_{2}, \cdots$ of closed intervals which are components of $N(X)$, such that (1) $K=\bigcup_{i=1}^{\infty} K_{i}$ is a closed subset of $N(X)$, (2) each $K_{i}$ is open in $K$, and (3) no subsequence of the $K_{i}$ 's converges to a point of $X$. Now any cover $\beta$ of $K$ can be refined by a cover $\alpha$ of $K$ such that for each $K_{i}, \alpha \cap K_{i}$ is a chain, each link of which is a proper subset of $K_{i}$. Since $K$ is a closed subset of $N(X)$, $q K \leqq q N(X) \leqq q(I \cup J)$; so there is a closed $\alpha$-map $f_{\alpha}$ of $K$ into $I \cup J$. But $f_{\alpha}[K] \cap J=\varnothing$, for otherwise $f_{\alpha}$ would map some $K_{i}$ into a point $p \in J$ and so, contrary to our assumption, $f_{\alpha}$ would not be an $\alpha$-map (since, for any open set $U$ about $p, f_{\alpha}^{-1}[U]$ would contain $K_{i}$ and therefore would not be contained in any element of $\alpha$.). So $f_{\alpha}$ is a closed $\alpha$-map of $K$ into $I$. This implies that $K$ is compact which is a contradiction.

THEOREM 2.10. If each component of $X$ is compact, then $q X \leqq q J^{*}$. 
Proof. If $X$ is totally disconnected, then $q X \leqq q J<q J^{*}$ and we are done. So assume otherwise and write $T=X-\bigcup_{i=1}^{n} C_{i}$ where the $C_{i}$ are the nondegenerate components of $X$ and $n \leqq \infty$. Let $\gamma$ be any covering of $X$. Since $\operatorname{dim} X \leqq 1, \gamma$ has a refinement $\gamma_{1}$ of order $\leqq 2$ (i.e., no 3 elements of $\gamma_{1}$ meet); say $\gamma_{1}$ consists of sets $W \cap X$ where $W$ is open in $R^{1}$. Let the components of the $W$ 's be denoted by $A_{i}$; they are open intervals, there is countably many of them, and no point of $X$ belongs to any 3 of them. The covering $\beta=\alpha \cap X$, where $\alpha=$ $\left\{A_{i} \mid i \in J\right\}$, refines $\gamma$. Denote $\alpha \cap C_{i}$ by $\alpha_{i}$. Let $\Delta$ be a denumerable dense subset of $R^{1}-X$ and let

$$
\begin{aligned}
& U=\left\{(a, b) \mid a, b \in \Delta, C_{i} \subset(a, b) \subset \bigcup\left\{A_{j} \mid A_{j} \in \alpha_{i}\right\} \text { for some } i(1 \leqq i \leqq n)\right\} \\
& \bigcup\left\{(a, b) \mid a, b \in \Delta, x \in(a, b) \subset A_{j} \text { for some } x \in T \text { and } A_{j} \in \alpha\right\} \text {. }
\end{aligned}
$$

Then since $\Delta$ is denumerable so is $U$ and we may write $U=\left\{U_{i} \mid i \in J\right\}$. Note that $U \cap X$ covers $X$.

Now we define the following:

(1) $\widetilde{U}_{1}=U_{1}, \widetilde{U}_{i}=U_{i}-\bigcup\left\{U_{j} \mid j=1, \cdots, i-1\right\}$ for $i=2,3, \cdots$,

(2) $\widetilde{U}=\left\{\widetilde{U}_{i} \mid \widetilde{U}_{i} \neq \varnothing, i \in J\right\}$,

(3) $\tilde{V}=\left\{\widetilde{K} \mid \widetilde{K}\right.$ is a component of some $\left.\widetilde{U}_{i} \in \widetilde{U}\right\}$.

Note that the elements of $\widetilde{V}$ are disjoint intervals (open, closed or half-open) having their end points in $\Delta$. So that $V=\widetilde{V} \cap X$ is a countable collection of disjoint, open and closed subsets of $X$ which covers $X$. Actually the elements of $V$ are strongly disjoint, i.e., if $K_{1}, K_{2} \in V$, then (glb $K_{1}$, lub $\left.K_{1}\right) \cap\left(\right.$ glb $K_{2}$, lub $\left.K_{2}\right)=\varnothing$.

We now index the elements of $V$ as follows: even positive integers are used to index the totally disconnected elements of $V$ and odd positive integers are used to index the elements of $V$ which are not totally disconnected. For any element $K_{i}$ of $V$, there are $a_{i}, b_{i} \in \Delta$, such that $K_{i}=\left(a_{i}, b_{i}\right) \cap X$. We now define a closed $\beta$-mapping, $f_{\beta}$, of $X$ into $J^{*}$ as follows:

(1) if $i$ is even, $f_{\beta}\left[K_{i}\right]=i$,

(2) if $i$ is odd, $\left(f_{\beta} \mid K_{i}\right)$ is an order preserving homeomorphism of $C_{j}=\left[c_{j}, d_{j}\right]$ (some nondegenerate component of $X$ contained in $K_{i}$ ) onto $[i, i+1 / 2] ; f_{\beta}\left[\left(a_{i}, c_{j}\right) \cap X\right]=i, f_{\beta}\left[\left(d_{j}, b_{i}\right) \cap X\right]=i+1 / 2$.

To show that $f_{\beta}$ is a $\beta$-mapping let:

$$
\begin{aligned}
\theta & =\bigcup\left\{f_{\beta}\left[K_{i}\right] \mid i=1,3,5, \cdots\right\}, \\
\lambda_{1} & =\{[i, i+1 / 2] \mid \theta \cap[i, i+1 / 2]=\varnothing\}, \\
\lambda_{2} & =\left\{f_{\beta}\left[\beta_{j} \cap K_{i}\right] \mid \beta_{j} \cap K_{i} \in \beta \cap K_{i} \text { and } i=1,3,5, \cdots\right\} .
\end{aligned}
$$

Then $\lambda=\lambda_{1} \cup \lambda_{2}$ is a covering of $J^{*}$. If $[i, i+1 / 2] \in \lambda_{1}$, then $f_{\beta}^{-1}[[i, i+1 / 2]]=K_{i}$ (or $\varnothing$ ) and $K_{i}$ is totally disconnected. By construction, $K_{i}$ is a subset of some $U_{h} \in U$, and either 


$$
C_{k} \subset U_{h} \subset \bigcup\left\{A_{j} \mid A_{j} \text { meets } C_{k}\right\}
$$

for some $C_{k}$, or $x \in U_{h} \subset A_{j}$ for some $x \in T$ and $A_{j} \in \alpha$. In the latter case, $K_{i}$ is contained in a single $A_{j}$. But this also follows in the other case, since otherwise some point of $X$ will belong to 3 distinct sets $A_{j}$. Thus $K_{i} \subset A_{j} \cap X \in \beta$. If $f_{\beta}\left[\beta_{j} \cap K_{i}\right] \in \lambda_{2}$, then $f_{\beta}^{-1}\left[f_{\beta}\left[\beta_{j} \cap K_{i}\right]\right] \subset \beta_{j} \in \beta$. Hence $f_{\beta}^{-1}[\lambda]>\beta$, i.e., $f$ is a $\beta$-mapping. Since $\beta>\gamma$ we have that $f_{\beta}$ is also a $\gamma$-mapping.

To show that $f_{\beta}$ is closed let $Z$ be a closed subset of $X$. Note that a set is closed in $J^{*}$ if and only if its intersection with each component of $J^{*}$ is closed. Since distinct $K_{i}$ 's are mapped into distinct components of $J^{*}$, it will suffice to prove that each $f_{\beta}\left[Z \cap K_{i}\right]$ is closed. If $i$ is even, $f_{\beta}\left[Z \cap K_{i}\right]$ has at most one point, so we need only consider the case when $i$ is odd. Since

$$
Z \cap K_{i}=\left(Z \cap C_{j}\right) \cup\left(Z \cap\left(K_{i}-C_{j}\right)\right)
$$

(where $C_{j}$ is the nondegenerate component of $K_{i}$ used in defining $f_{\beta}$ ), we can write

$$
f_{\beta}\left[Z \cap K_{i}\right]=f_{\beta}\left[Z \cap C_{j}\right] \cup f_{\beta}\left[Z \cap\left(K_{i}-C_{j}\right)\right] .
$$

Then, since $Z \cap C_{j}$ is closed in $X$ and $f_{\beta}$ is a homeomorphism on $C_{j}$, we have that $f_{\beta}\left[Z \cap C_{j}\right]$ is a closed subset of $[i, i+1 / 2]$. Furthermore, $f_{\beta}\left[Z \cap\left(K_{i}-C_{j}\right)\right] \subset\{i\} \cup\{i+1 / 2\}$ and hence is also closed. Therefore, we may conclude that $f_{\beta}\left[Z \cap K_{i}\right]$ is a closed subset of $[i, i+1 / 2]$ when $i$ is odd. So that $f_{\beta}[Z] \cap[i, i+1 / 2]$ is closed, for each $i \in J$, and hence $f_{\beta}[Z]$ is a closed subset of $J^{*}$.

Thus, for each covering $\gamma$ of $X$, we have shown the existence of a closed $\gamma$-mapping of $X$ into $J^{*}$. Therefore, we have the desired result, i.e., $q X \leqq q J^{*}$.

\section{THEOREM 2.11. $q(I \cup J) \ll q J^{*}$.}

Proof. Let $\alpha$ be a covering of $I \cup J$. Then a map which is a homeomorphism of $I$ onto [1,3/2] and takes $j \in J$ into $j+1$ is a closed $\alpha$-map of $I \cup J$ into $J^{*}$. So $q(I \cup J) \leqq q J^{*}$. Now suppose $q J^{*} \leqq$ $q(I \cup J)$. Then Lemma 2.1 implies that $q J^{*} \leqq q I$. But this is impossible by Theorem 1.1. So $q(I \cup J)<q J^{*}$.

Suppose there is a set $X$ such that $q(I \cup J)<q X<q J^{*}$. Then by Theorem $1.1 X$ is not compact and not totally disconnected. In addition, each component of $X$ is compact (by Corollary 1.1). Suppose $N(X)$ is not compact. Then there is a sequence $K_{1}, K_{2}, \cdots$ of closed intervals which are components of $N(X)$, such that (1) $K=\bigcup_{i=1}^{\infty} K_{i}$ is a closed subset of $N(X),(2)$ each $K_{i}$ is open in $K$, and (3) no 
subsequence of the $K_{i}$ 's converges to a point of $X$. Since there is a homeomorphism of $J^{*}$ onto $K$ (just send $[i,(i+1) / 2]$ homeomorphically onto $K_{i}$, for each $i \in J$ ) and $K$ is closed it $X$, it follows that $q J^{*} \leqq$ $q X$. This contradicts our assumption that $q X<q J^{*}$.

Now suppose $N(X)$ is compact. Let $\gamma$ be any covering of $X$. Proceeding as in the proof of Theorem 2.10 one obtains a closed $\beta$ map $f_{\beta}$ of $X$ into $J^{*}$ (where $\beta>\gamma$ ). Under this mapping the image of $N(X)$ is the union of a finite number of closed intervals which are components of $J^{*}$. We follow $f_{\beta}$ by a homeomorphism $g_{\beta}$ of $f_{\beta}[X]$ onto a closed subset of $I \cup J$. The homeomorphism $g_{\beta}$ can be defined as one which takes $f_{\beta}[N(X)]$ homeomorphically into $I$ and $f_{\beta}[X]-f_{\beta}[N(X)]$ homeomorphically into $J$. Consequently, $q X \leqq q(I \cup J)$ which contradicts our assumption that $q(I \cup J)<q X$. So this together with the previous paragraph imply that $q(I \cup J) \ll q J^{*}$.

Lemma 2.2. If $X$ is not compact and not totally disconnected, then $q(I \cup J) \leqq q X$.

Proof. If $X$ contains a component $K$ which is an open or halfopen interval, then $q(I \cup J) \leqq q K \leqq q X$ and we are done. Assume now that the components of $X$ are compact and that $K$ is one such nondegenerate component. Since $X$ is not compact, it has a closed subset $Z$ which is homeomorphic to $J$ and disjoint from $K$. Now we claim that the hypotheses of Theorem 1.2 are satisfied, for (1) $q I \leqq$ $q K$, (2) $q J \leqq q Z$, (3) $I$ and $J$ are disjoint closed subsets of $I \cup J$; (4) $K$ and $Z$ are disjoint closed subsets of $K \cup Z$. So applying Theorem 1.2 we conclude that $q(I \cup J) \leqq q(K \cup Z) \leqq q X$.

Theorem 2.12. $q X=q(I \cup J)\left(q X=q J^{*}\right)$ if and only if (1) $X$ is not compact, (2) not totally disconnected, (3) each of its components is compact, and (4) $N(X)$ is (is not) compact.

Proof. Assume that conditions (1)-(4) hold. As consequences of Theorem 2.10 and Lemma 2.2 we have $q(I \cup J) \leqq q X \leqq q J^{*}$. So since $q(I \cup J)$ immediately precedes $q J^{*}$ we have that $q X$ equals one or the other. Now if $N(X)$ is compact, it follows from Theorem 1.1 that $q J^{*} \geqq q X$. Hence in this case we have that $q X=q(I \cup J)$. Now consider the other case, i.e., when $N(X)$ is not compact. Suppose $q X \leqq q(I \cup J)$. Then as a result of Lemma 2.1 we have that $q N(X) \leqq q I$. However, this imlpies that $N(X)$ is compact contrary to our assumption. Hence in this case $q X=q J^{*}$.

Now assume that $q X=q(I \cup J)$ or $q X=q J^{*}$. Then in both cases by Theorem 1.1 and Corollary 1.1, we have that: (1) $X$ is not compact, 
(2) not totally disconnected and (3) each component of $X$ is compact. In the former case, as a result of Lemma 2.1 we have that $q N(X) \leqq$ $q I$ and hence $N(X)$ is compact. In the latter case, suppose $N(X)$ is compact. Then, by the first part of this proof $q X=q(I \cup J)$. But $q(I \cup J)<q J^{*}$ so we have a contradiction. Thus in this case $N(X)$ is not compact.

\section{THEOREM 2.13. $q J^{*} \ll q L_{1}$.}

Proof. First, $q J^{*} \leqq q L_{1}$ since the function $f$ defined by $f(x)=$ $(1-5 x) / 2 x$ is a homeomorphism of $J^{*}$ onto a closed subset of $L_{1}$. Since $L_{1}$ is connected and not compact, Corollary 1.1 implies that $q L_{1} \not \leq q J^{*}$ and therefore $q J^{*}<q L_{1}$. Now suppose there is a set $X$ such that $q J^{*}<q X<q L_{1}$. Then $X$ is not compact and some component of $X$ is not compact (a consequence of Theorem 2.10). Let $K$ denote such a component. Then $q L_{1} \leqq q K \leqq q X$ which is a contradiction and so $q J^{*} \ll q L_{1}$.

LeMma 2.3. If $q X \leqq q Y$, then $X$ does not have more components than $Y$ which are open intervals.

Proof. First we will show that $q M_{1} \not L_{1}$. Suppose not, i.e., $q M_{1} \leqq q L_{1}$. Then, for any covering $\alpha$ of $M_{1}$, there exists a closed $\alpha$ mapping, $f_{\alpha}$, of $M_{1}$ into $L_{1}$. Let $S=(-3 / 2,-11 / 8], T=[-9 / 8,-1)$ and $\mathscr{A}$ be the collection of coverings of $M_{1}$ with the property that none of their elements intersects both $S$ and $T$. (Note that $\mathscr{A}$ is cofinal in the collection of all coverings of $M_{1}$.) For any sufficiently fine $\alpha \in \mathscr{A}$, there are points $y_{1}, y_{2} \in L_{1}$ such that $f_{\alpha}[S]=\left(-5 / 2, y_{1}\right]$ and $f_{\alpha}[T]=\left(-5 / 2, y_{2}\right]$. This is the case since each image set is closed in $L_{1}$, connected and not compact (for $\alpha$ sufficiently fine). But this implies $f_{\alpha}^{-1}\left(\min \left(y_{1}, y_{2}\right)\right)$ intersects both $S$ and $T$ which contradicts the fact that $f_{\alpha}$ is an $\alpha$-mapping. Therefore, $q M_{1} \not \$ q L_{1}$.

We now show, for any sufficiently fine $\alpha \in \mathscr{A}$, that a closed $\alpha$ mapping, $f_{\alpha}$, of $M_{1}$ into itself actually takes $M_{1}$ onto itself. Suppose not, i.e., for $\alpha \in \mathscr{B}$, a cofinal subset of $\mathscr{A}, f_{\alpha}\left[M_{1}\right]$ is a proper subset of $M_{1}$. Then, for $\alpha$ sufficiently fine, $f_{\alpha}\left[M_{1}\right]=\left(-3 / 2, y_{\alpha}\right]$ or $\left[y_{\alpha},-1\right)$ where $y_{\alpha} \in M_{1}$, since $f_{\alpha}\left[M_{1}\right]$ is closed in $M_{1}$, connected and not compact. We consider only the first of the two cases since they are similar. Let $g_{\alpha}$ be a homeomorphism of $\left(-3 / 2, y_{\alpha}\right]$ onto $L_{1}$. Then, for any $\alpha \in \mathscr{B}, g_{\alpha} f_{\alpha}$ is a closed $\alpha$-mapping of $M_{1}$ into $L_{1}$. But this is impossible since $q M_{1} \not \geqq q L_{1}$. Hence, for sufficiently fine $\alpha \in \mathscr{A}, f_{\alpha}\left[M_{1}\right]=M_{1}$.

We now prove that if $X$ has at least $n$ components which are open intervals, then so does $Y$, for $n \in J$. Let $\mathscr{K}$ denote the collection of components of $X$ which are open intervals. Let $\alpha$ be any 
covering of $X$ such that:

(1) if $K_{i} \in \mathscr{K}$, then $\alpha \cap K_{i}$ is a covering of $K_{i}$ corresponding to a covering of $M_{1}$ in $\mathscr{A}$, under the obvious linear homeomorphism of $K_{i}$ onto $M_{1}$,

(2) if $A \in \alpha$, then $A$ intersects at most one $K_{i} \in \mathscr{K}$. Then, for $\alpha$ sufficiently fine and a closed $\alpha$-mapping, $f_{\alpha}$, we have that (1) implies $f_{\alpha}\left[K_{i}\right]=C_{i}$, a component of $Y$ which is an open interval. This is the case since $f_{\alpha}\left[K_{i}\right]$ is not contained in a component of $Y$ which is a half-open interval (otherwise $q M_{1} \leqq q L_{1}$ ), nor in one which is a closed interval (otherwise $K_{i}$ is compact) and so $f_{\alpha}\left[K_{i}\right]$ must be contained in one which is an open interval. But we have already shown in this situation that $\left(f_{\alpha} \mid K_{i}\right)$ maps $K_{i}$ onto this open interval (for $\alpha$ sufficiently fine). Furthermore, since (2) implies $C_{i} \cap C_{j}=\varnothing$, for $i \neq j$, $Y$ has at least $n$ components which are open intervals.

Lemma 2.4. If $q X \leqq q\left(L_{1} \cup J^{*}\right)$, then $X$ has at most one component which is a half-open interval.

Proof. Suppose $X$ has two components which are half-open intervals, say $K_{1}$ and $K_{2}$. Let $\alpha$ be a covering of $X$ in which no element intersects both $K_{1}$ and $K_{2}$. Since $q\left(K_{1} \cup K_{2}\right) \leqq q X \leqq q\left(L_{1} \cup J^{*}\right)$ there is a closed $\beta$-mapping $f_{\beta}$ of $K_{1} \cup K_{2}$ into $L_{1} \cup J^{*}$ (where $\beta=\alpha \cap\left(K_{1} \cup K_{2}\right)$ ). It follows from the fact that $f_{\beta}\left[K_{1}\right]$ and $f_{\beta}\left[K_{2}\right]$ are closed, connected and not compact (for sufficiently fine $\beta$ ), that $f_{\beta}\left[K_{1} \cup K_{2}\right] \subset L_{1}$. Furthermore, there are points $y_{1}, y_{2}$ of $L_{1}$ such that $f_{\beta}\left[K_{1}\right]=\left(-5 / 2, y_{1}\right]$ and $f_{\beta}\left[K_{2}\right]=\left(-5 / 2, y_{2}\right]$ since each image set is closed in $L_{1}$, connected and not compact (for $\beta$ sufficiently fine). But this implies that $f_{\beta}^{-1}\left(\min \left(y_{1}, y_{1}\right)\right)$ intersects both $K_{1}$ and $K_{2}$ which contradicts the fact that $f_{\beta}$ is a $\beta$-map. Therefore, $X$ does not have two components which are half-open intervals.

LEMmA 2.5. If $q L_{1} \leqq q X \leqq q\left(L_{1} \cup J^{*}\right)$, then $X$ has exactly one component which is a half-open interval and none which is an open interval.

Proof. By Lemma $2.3 X$ does not have a component which is an open interval. Suppose each component of $X$ is compact. Then Theorem 2.10 implies that $q X \leqq q J^{*}$. But $q J^{*}<q L_{1}$ and we have a contradiction. So $X$ has at least one component which is not compact and none which is an open interval. Hence there is at least one component of $X$ which is a half-open interval. Finally, Lemma 2.4 implies that $X$ has exactly one component which is a half-open interval.

THEOREM 2.14. $q X=q L_{1}$ if and only if (1) exactly one of the 
components of $X$ is a half-open interval, (2) this component, say $K$, has the property that the closure (in $X$ ) of $X-K$ is compact.

Proof. We first assume that $q X=q L_{1}$. Then $X$ can not be compact, nor totally disconnected. By Lemma $2.5 X$ has exactly one component, say $K$, which is a half-open interval and none which is an open interval. Let $Y=\mathrm{Cl}(X-K)$ (we assume $Y \neq \varnothing$ ) and let $\beta$ be any covering of $Y$. Then $\beta$ can be extended to a covering $\alpha$ of $X$ (i.e., $\alpha \cap Y=\beta$ and $\alpha$ is a covering of $X$ ). Since $q X \leqq q L_{1}$ there is a closed $\alpha$-map, $f_{\alpha}$, of $X$ into $L_{1}$. Moreover, since $f_{\alpha}[K]$ is closed in $L_{1}$, connected and not compact (for $\alpha$ sufficiently fine), $f_{\alpha}[K]$ must be of the form $(-5 / 2, c]$ where $-5 / 2<c \leqq-2$. Let $g_{\beta}=\left(f_{\alpha} \mid Y\right)$ and consider $\beta$ to be fine enough so that $d=\operatorname{glb}\left(g_{\beta}[Y]\right)>-5 / 2$. Then $g_{\beta}$ is a closed $\beta$-map of $Y$ into $L_{1}-(-5 / 2, d)$, a compact set. Thus, for any sufficiently fine covering $\beta$, we have $g_{\beta}[Y]$ is compact and so $Y$ is compact.

We now consider the converse. Since $X$ has a component $K$ which is a half-open interval, we have that $q L_{1}=q K \leqq q X$. Now assume $X-K \neq \varnothing$. Then there are disjoint closed subsets $X_{1}$ and $X_{2}$ of $X$ such that $X=X_{1} \cup X_{2}, X_{1} \supset K, X_{1}$ is homeomorphic to a closed subset of $(-5 / 2,-9 / 4]$ and $X_{2}$ is homeomorphic to a compact subset of $(-9 / 4,-2]$. Hence by Theorem 1.2 we have that $q X \leqq q L_{1}$.

THEorem 2.15. $q L_{1} \ll q\left(L_{1} \cup J\right)$.

Proof. Since $L_{1}$ is closed in $L_{1} \cup J$ we have $q L_{1} \leqq q\left(L_{1} \cup J\right)$. As a consequence of Theorem 2.14 we have $q\left(L_{1} \cup J\right) \neq q L_{1}$ and so $q L_{1}<q\left(L_{1} \cup J\right)$. Suppose there is a set $X$ such that $q L_{1}<q X<q\left(L_{1} \cup J\right)$. Then $X$ cannot be compact nor totally disconnected. Moreover, Lemma 2.5 implies that $X$ has exactly one component, say $K$, which is a halfopen interval and none which is an open interval. So $\mathrm{Cl}(X-K)$ is not compact (otherwise $\left.q L_{1}=q X\right)$. Since $\mathrm{Cl}(X-K)$ is not compact it contains a closed subset $Z$ homeomorphic to $J$. Therefore $q\left(L_{1} \cup J\right) \leqq$ $q(K \cup Z) \leqq q X$. This is a contradiction.

\section{LEMMA 2.6. $q\left(L_{1} \cup J\right)<q\left(L_{1} \cup J^{*}\right)$.}

Proof. Since $q J \leqq q J^{*}$, by Theorem 1.2 we have that $q\left(L_{1} \cup J\right) \leqq$ $q\left(L_{1} \cup J^{*}\right)$. Suppose $q\left(L_{1} \cup J^{*}\right) \leqq q\left(L_{1} \cup J\right)$. Any cover $\beta$ of $L_{1} \cup J^{*}$ can be refined by a subcover $\alpha$ such that, for each component $K$ of $L_{1} \cup J^{*}, \alpha \cap K$ is a chain, each link of which is a proper subset of $K$. By assumption, there is a closed $\alpha$-map $f_{\alpha}$ of $L_{1} \cup J^{*}$ into $L_{1} \cup J$. But $f_{\alpha}\left[L_{1} \cup J^{*}\right] \cap J=\varnothing$, for otherwise $f_{\alpha}$ would map some component $K$ of $L_{1} \cup J^{*}$ into a point $p \in J$ and so, contrary to our assumption, $f_{\alpha}$ would not be an $\alpha$-map (since, for any open set $U$ about $p, f_{\alpha}^{-1}[U]$ 
would contain $K$ and therefore would not be contained in any element of $\alpha$ ). So $f_{\alpha}$ is a closed $\alpha$-map of $L_{1} \cup J^{*}$ into $L_{1}$, which implies $q\left(L_{1} \cup J^{*}\right) \leqq q L_{1}$. But then $q\left(L_{1} \cup J^{*}\right)=q L_{1}$ and this contradicts Theorem 2.14. Therefore, $q\left(L_{1} \cup J^{*}\right) \npreceq q\left(L_{1} \cup J\right)$ and we have $q\left(L_{1} \cup J\right)<$ $q\left(L_{1} \cup J^{*}\right)$.

Lemma 2.7. If $X$ contains exactly one component $K$ which is a half-open interval, none which is an open interval, and $N(X-K)$ is not compact, then $q\left(L_{1} \cup J^{*}\right) \leqq q X$.

Proof. Since $N(X-K)$ is not compact, it contains a sequence $K_{1}, K_{2}, \cdots$ of nondegenerate components of $X$ which are closed intervals and no subsequence of $K_{1}, K_{2}, \cdots$ converges to a point of $X$. Therefore, $\bigcup_{i=1}^{\infty} K_{i}$ is a closed subset of $X$ which is homeomorphic to $J^{*}$. As $K$ is also closed in $X, K \cup\left(\bigcup_{i=1}^{\infty} K_{i}\right)$ is a closed subset of $X$ homeomorphic to $L_{1} \cup J^{*}$. Hence $q\left(L_{1} \cup J^{*}\right) \leqq q X$.

THEOREM 2.16. $q X=q\left(L_{1} \cup J\right)$ if and only if (1) exactly one of the components of $X$ is a half-open interval, (2) this component, say $K$, has property that the closure (in $X$ ) of $X-K$ is not compact, and (3) $N(X-K)$ is compact.

Proof. Assume that $X$ satisfies conditions (1), (2) and (3). First we will show that $q\left(L_{1} \cup J\right) \leqq q X$. Since $\mathrm{Cl}(X-K)$ (in $X$ ) is not compact, it contains a closed subset $Z$ which is homeomorphic to $J$. Thus each subset of $Z$ is closed in $Z$ and, since $Z$ is closed in $X$, each subset of $Z$ is closed in $X$. Furthermore, $\mathrm{Cl}(X-K)=X-K$ or $(X-K) \cup\{p\}$, where $p$ is the end point of $K$ which is in $K$. Therefore, $Y=Z-p$ is closed in $X, Y \subset X-K$ and $Y$ is homeomorphic to $J$. As $K$ is also closed in $X, K \cup Y$ is a closed subset of $X$ homeomorphic to $L_{1} \cup J$. Therefore we have $q\left(L_{1} \cup J\right) \leqq q X$.

Now we show that $q X \leqq q\left(L_{1} \cup J\right)$. Let $\gamma$ be any covering of $X$ and $K=(c, d]$. Then $\gamma$ can be refined by a covering $\beta=\alpha \cap X$, where $\alpha=\left\{A_{j} \mid j \in J\right\}$ is a collection of open intervals such that: (1) $\bigcup_{j=1}^{\infty} A_{j} \supset X$, (2) if $A_{j} \in \alpha_{0}=\alpha \cap K$, then $c \notin A_{j}$ and (3) there is exactly one $A_{j} \in \alpha_{0}$ such that $d \in A_{j}$. Let $B=(c, b)$ where

$$
b \in\left[\left(R^{1}-X\right) \cap\left(\cup\left\{A_{j} \mid A_{j} \in \alpha_{0}\right\}\right)\right] .
$$

Then $W=B \cap X$ is open and closed in $X$, and $K \subset W \subset \bigcup\left\{A_{j} \mid A_{j} \in \alpha_{0}\right\}$. So $W$ and $Y=X-W$ are closed disjoint subsets of $X$ whose union is $X$. Moreover, a mapping $f$, which is a homeomorphism of $K$ onto $(-5 / 2,-9 / 4]$ and takes $W-K$ into the point $-9 / 4$, is a closed ( $\beta \cap W)$-mapping of $W$ into $(-5 / 2,-9 / 4]$. Furthermore, since $\beta>\gamma$ 
we have that $f$ is also a $(\gamma \cap W)$-mapping of $W$ into $(-5 / 2,-9 / 4]$.

Now we consider $Y$. Case 1: $Y$ is compact or totally disconnected, Then $q Y \leqq q(I \cup J)$. Case 2: $\quad Y$ is neither compact nor totally disconnected. Then it follows from condition (3) of our hypothesis that $N(Y)$ and each comporent of $Y$ are compact. Therefore, Theorem 2.12 applies and we have that $q Y \leqq q(I \cup J)$. So, in both cases, we have $q Y \leqq q(I \cup J)=q([-17 / 8,-2] \cup J)$. Thus there is a closed $(\gamma \cap Y)$ mapping, $g$, of $Y$ into $[-17 / 8,-2] \cup J$. Therefore, the mapping which is $f$ on $W$ and $g$ on $Y$ is a closed $\gamma$-mapping of $X$ into $L_{1} \cup J$. Thus $q X \leqq q\left(L_{1} \cup J\right)$ and since we already have shown $q\left(L_{1} \cup J\right) \leqq q X$ it follows that $q X=q\left(L_{1} \cup J\right)$.

Now we assume that $q X=q\left(L_{1} \cup J\right)$. Then $X$ is not compact nor totally.disconnected. By Lemma 2.6 we have that $q\left(L_{1} \cup J\right) \leqq q\left(L_{1} \cup J^{*}\right)$. So Lemma 2.5 applies and $X$ has exactly one component, say $K$, which is a half-open interval and none which is an open interval. So all components of $X$, other than $K$, are compact. As a consequence of Theorems 2.14 and 2.15 we have that $\mathrm{Cl}(X-K)$ is not compact and so neither is $X-K$. Now $N(X-K)$ is compact, otherwise by Lemma $2.7 q\left(L_{1} \cup J^{*}\right) \leqq q X$ which is impossible by Lemma 2.6.

TheOREM 2.17. $q\left(L_{1} \cup J\right) \ll q\left(L_{1} \cup J^{*}\right)$.

Proof. By Lemma 2.6 we have that $q\left(L_{1} \cup J\right)<q\left(L_{1} \cup J^{*}\right)$. Now suppose there is a set $X$ such that $q\left(L_{1} \cup J\right)<q X<q\left(L_{1} \cup J^{*}\right)$. Then by Lemma $2.5 X$ has exactly one component, say $K$, which is a halfopen interval and none which is an open interval. Now $N(X-K)$ is not compact, otherwise Theorems 2.14 and 2.16 would imply $q X \leqq$ $q\left(L_{1} \cup J\right)$. But then Lemma 2.7 implies that $q\left(L_{1} \cup J^{*}\right) \leqq q X$ which is a contradiction.

Theorem 2.18. $q X=q\left(L_{1} \cup J^{*}\right)$ if and only if (1) exactly one of the components of $X$, say $K$, is a half-open interval, (2) no component of $X$ is an open interval, and (3) $N(X-K)$ is not compact.

Proof. Assume $X$ satisfies conditions (1), (2) and (3). Then by Lemma 2.7 we have $q\left(L_{1} \cup J^{*}\right) \leqq q X$. Next we show that $q X \leqq$ $q\left(L_{1} \cup J^{*}\right)$. Let $\gamma$ be any covering of $X$ and $K=(c, d]$. Then, as in the proof of Theorem 2.16, we have $X=W \cup Y$, where $W, Y$ are closed disjoint subsets of $X$ and $W \subset K$. Moreover, there is a closed ( $\gamma \cap W$ )-mapping, $f$, of $W$ into $L_{1}$, and $q Y \leqq q J^{*}$ (since each component of $Y$ is compact and Theorem 2.10 applies). Thus there is a closed $\left(\gamma \cap Y\right.$ )-mapping, $g$, of $Y$ into $J^{*}$. So the mapping which is $f$ on $W$ and $g$ on $Y$ is a closed $\gamma$-mapping of $X$ into $L_{1} \cup J^{*}$. Therefore, we have $q X \leqq q\left(L_{1} \cup J^{*}\right)$ and so $q X=q\left(L_{1} \cup J^{*}\right)$. 
Assume now that $q X=q\left(L_{1} \cup J^{*}\right)$. Then Lemma 2.5 implies that $X$ has exactly one component, say $K$, which is a half-open interval and none.which is an open interval. If $\mathrm{Cl}(X-K)$ is compact, then Theorem 2.14 implies $q X=q L_{1}$. This is a contradiction to our assumption that $q X=q\left(L_{1} \cup J^{*}\right)$ since $q L_{1}<q\left(L_{1} \cup J^{*}\right)$. So we now assume that $\mathrm{Cl}(X-K)$ is not compact. Then, if $N(X-K)$ is compact, Theorem 2.16 implies that $q X=q\left(L_{1} \cup J\right)$. This is impossible so $N(X-K)$ is not compact.

Lemma 2.8. If $q Y \leqq q X$ and $X$ does not have a component which is an open interval, then $Y$ does not have more components than $X$ which are half-open intervals.

Proof. We prove that if $Y$ has at least $n$ components which are half-open intervals, then so does $X$, for each $n \in J$. Let $K_{1}, K_{2}, \cdots, K_{n}$ denote $n$ of these components in $Y$. Suppose $X$ has only $m<n$ components which are half-open intervals, say $C_{1}, \cdots, C_{m}$. For any covering $\alpha$ of $Y$, let $f_{\alpha}$ be a closed $\alpha$-mapping of $Y$ into $X$. Since the components of $X$, other than the $C_{i}$, are compact, it follows that $f_{\alpha}\left[\bigcup_{i=1}^{n} K_{i}\right] \subset \bigcup_{i=1}^{m} C_{i}$, for $\alpha$ sufficiently fine. Therefore, some two $K_{i}$, say $K_{1}$ and $K_{2}$, are such that $f_{\alpha}\left[K_{1} \cup K_{2}\right]$ is contained in some one $C_{i}$. This implies that $q L_{2} \leqq q L_{1}$ which contradicts Lemma 2.4. Hence, $X$ has at least $n$ components which are half-open intervals.

\section{TheOREM 2.19. $q\left(L_{1} \cup J^{*}\right) \ll q L_{2}$.}

Proof. It follows from Theorem 1.2 that $q\left(L_{1} \cup J^{*}\right) \leqq q L_{2}$ and from Lemma 2.4 that $q L_{2} \leqq q\left(L_{1} \cup J^{*}\right)$. Thus $q\left(L_{1} \cup J^{*}\right)<q L_{i}$. Now suppose there is a set $X$ such that $q\left(L_{1} \cup J^{*}\right)<q X<q L_{2}$. Then, by Lemma 2.3, $X$ does not have a component which is an open interval. Applying Lemma 2.8 to $q\left(L_{1} \cup J^{*}\right)<q X$ we conclude that $X$ has at least one component which is a half-open interval. On the other hand, $X$ has fewer than two such components (otherwise $q L_{2} \leqq q X$ ). Therefore, $X$ has exactly one component which is a half-open interval. So $X$ satisfies the conditions of Theorem $2.14,2.16$ or 2.18 and we may conclude that $q X \leqq q L_{1}, q X \leqq q\left(L_{1} \cup J\right)$ or $q X \leqq q\left(L_{1} \cup J^{*}\right)$. Hence, we have that $q X \leqq q\left(L_{1} \cup J^{*}\right)$. But this is a contradiction and we have that $q\left(L_{1} \cup J^{*}\right) \ll q L_{2}$.

Theorem 2.20. $q X=q L_{n}(n \in J)$ if and only if $X$ has exactly $n$ components which are half-open intervals, say $K_{1}, \cdots, K_{n}$, and the closure (in $X)$ of $X-\bigcup_{i=1}^{n} K_{i}$ is compact.

Proof. We first assume that $q X=q L_{n}$. Then, by Lemma 2.3, $X$ 
does not have a component which is an open interval. So Lemma 2.8 applies (twice) and $X$ has exactly $n$ components which are half-open intervals, say $K_{1}, \cdots, K_{n}$. Let $K=\bigcup_{i=1}^{n} K_{i}, Y=\mathrm{Cl}(X-K)$ (we assume $Y \neq \varnothing)$ and let $\beta$ be any covering of $Y$. Then $\beta$ can be extended to a covering $\alpha$ of $X$ (i.e., $\alpha \cap X=\beta$ and $\alpha$ is a covering of $X$ ). Since $q X \leqq q L_{n}$ there is a closed $\alpha$-mapping, $f_{\alpha}$, of $X$ into $L_{n}$. Moreover, since $f_{\alpha}[K]$ is closed in $L_{n}$ and, for $\alpha$ sufficiently fine, each of its components is not compact, $f_{\alpha}[K]=\bigcup\left\{\left(-2 j-1 / 2, c_{j}\right] \mid j \in J_{n}\right.$, where $\left.-2 j-1 / 2<c_{j} \leqq-2 j\right\}$. Let $g_{\beta}=\left(f_{\alpha} \mid Y\right)$ and consider $\beta$ to be fine enough so that $d_{j}=\operatorname{glb}\left(g_{\beta}[Y] \cap(-2 j-1 / 2,-2 j]\right)>-2 j-1 / 2$, for $j \in J_{n}$. Then $g_{\beta}$ is a closed $\beta$-mapping of $Y$ into

$$
L_{n}-\bigcup\left\{\left(-5 / 2, d_{j}\right) \mid j \in J_{n}\right\},
$$

a compact set. Thus, for any sufficiently fine covering $\beta$, we have $g_{\beta}[Y]$ is compact and so $Y$ is compact.

Now consider the converse. So $X$ has $n$ components, $K_{1}, \cdots, K_{n}$, which are half-open intervals. Let $K=\bigcup_{i=1}^{n} K_{i}$. Then we have $q L_{n}=q K \leqq q X$. Now assume $X-K \neq \varnothing$. Then there are disjoint closed subsets, $X_{1}$ and $X_{i}$, of $X$ such that $X=X_{1} \cup X_{2}, X_{1} \supset K, X_{1}$ is homeomorphic to a closed subset of $\bigcup\left\{(-2 j-1 / 2,-2 j-1 / 4] \mid j \in J_{n}\right\}$ and $X_{2}$ is homeomorphic to a compact subset of

$$
\mathbf{U}\left\{(-2 j-1 / 4,-2 j] \mid j \in J_{n}\right\} \text {. }
$$

Hence, by Theorem 1.2 we have $q X \leqq q L_{n}$.

THEOREM 2.21. $q L_{n} \ll q\left(L_{n} \cup J\right), n \in J$.

Proof. Since $L_{n}$ is closed in $L_{n} \cup J$ we have $q L_{n} \leqq\left(L_{n} \cup J\right)$. As a consequence of Theorem 2.20 we have $q\left(L_{n} \cup J\right) \neq q L_{n}$ and so $q L_{n}<q\left(L_{n} \cup J\right)$. Suppose there is a set $X$ such that

$$
q L_{n}<q X<q\left(L_{n} \cup J\right) .
$$

Then Lemma 2.3 implies $X$ does not have a component which is an open interval. So Lemma 2.8 can be applied (twice) to get that $X$ has exactly $n$ components, say $K_{1}, \cdots, K_{n}$, which are half-open intervals. Let $K=\bigcup_{i=1}^{n} K_{i}$. Then $\mathrm{Cl}(X-K)$ is not compact (otherwise $q L_{n}=$ $q X)$. Therefore $\mathrm{Cl}(X-K)$ contains a closed subset $Z$ homeomorphic to $J$ and so $q\left(L_{n} \cup J\right) \leqq q(K \cup Z) \leqq q X$. This is a contradiction.

The next four theorems have proofs similar to those of Theorems 2.16-2.19 and therefore omitted.

Theorem 2.22. $q X=q\left(L_{n} \cup J\right)(n \in J)$ if and only if (1) $X$ has exactly $n$ components which are half-open intervals, say $K_{1}, \cdots, K_{n}$, 
(2) the closure (in $X$ ) of $X-\bigcup_{i=1}^{n} K_{i}$ is not compact and (3) $N\left(X-\bigcup_{i=1}^{n} K_{i}\right)$ is compact.

Theorem 2.23. $q\left(L_{n} \cup J\right) \ll q\left(L_{n} \cup J^{*}\right), n \in J$.

Theorem 2.24. $q X=q\left(L_{n} \cup J^{*}\right)(n \in J)$ if and only if (1) $X$ has exactly $n$ components which are half-open intervals, say $K_{1}, \cdots, K_{n}$, (2) no component of $X$ is an open interval and (3) $N\left(X-\bigcup_{i=1}^{n} K_{i}\right)$ is not compact.

Theorem 2.25. $q\left(L_{n} \cup J^{*}\right) \ll q L_{n+1}, n \in J$.

Theorem 2.26. $q L_{n} \leqq q X<q L_{n+1}(n \in J)$ if and only if $X$ has exactly $n$ components which are half-open interval and none which is an open interval.

Proof. Assume $q L_{n} \leqq q X<q L_{n+1}$. Then, by Theorems 2.21, 2.23, and 2.25, we have $q X=q L_{n}, q X=q\left(L_{n} \cup J\right)$ or $q X=q\left(L_{n} \cup J^{*}\right)$. So, by Theorem $2.20,2.22$ and 2.24 , we have $X$ has exactly $n$ components which are half-open intervals and none which is an open interval.

Now assume that $X$ has exactly $n$ components which are half-open intervals and none which is an open interval. By Theorems 2.20,2.22 and 2.24 we have that $q X=q L_{n}, q X=q\left(L_{n} \cup J\right)$ or $q X=q\left(L_{n} \cup J^{*}\right)$. Hence, by Theorems 2.21, 2.23 and 2.25 we have $q L_{n} \leqq q X<q L_{n+1}$.

THEOREM 2.27. $q L^{*} \ll q L_{\infty}$.

Proof. Any covering $\alpha$ of $L^{*}$ can be refined by a covering $\beta=$ $\left\{B_{j} \mid j=0,1,2, \cdots\right\}$ such that:

(1) $B_{0}=[0,1 /(2 m+1)) \cap L^{*}$ for some $m \in J$,

(2) $B_{0} \cap B_{j}=\varnothing$ for $B_{j} \in \beta$ and $j \neq 0$.

Since $B=B_{0}$ is open and closed in $L^{*}$ so is $Y=\bigcup_{j=1}^{\infty} B_{j}$. Moreover, $Y$ and $B$ are disjoint, $L^{*}=Y \cup B$ and

$$
Y=\bigcup\{[1 /(i+1), 1 / i) \mid i=1,3, \cdots, 2 m-1\} .
$$

We now define a closed $\beta$-mapping, $f_{\beta}$, of $L^{*}$ into $L_{-m}$ (a closed subset of $\left.L_{\infty}\right)$. Let $f_{\beta}[B]=-4 m$ and $\left(f_{\beta} \mid[1 /(i+i), 1 / i)\right)$ be a homeomorphism of $[1 /(i+1), 1 / i)$ onto $(-2 i-1 / 2,-2 i]$, for $i=1,3, \cdots, 2 m-1$. Since $\beta>\alpha$ we have that $f_{\beta}$ is a closed $\alpha$-mapping of $L^{*}$ into $L_{\infty}$. Therefore, we may conclude that $q L^{*} \leqq q L_{\infty}$.

Now suppose that $q L_{\infty} \leqq q L^{*}$. Then, for any covering $\alpha$ of $L_{\infty}$, there is a closed $\alpha$-mapping, $f_{\alpha}$ of $L_{\infty}$ into $L^{*}$. We can write each component of $L_{\infty}$ as the union of at least two distinct subsets which 
are open in $L_{\infty}$. Let $\alpha$ be any covering of $L_{\infty}$ of this type. Now Lemma 2.8 implies that $f_{\alpha}$ takes distinct components of $L_{\infty}$ into distinct components of $L^{*}$, for $\alpha$ sufficiently fine. So $f_{\alpha}\left[L_{\infty}\right]$ must contain a sequence of distinct components of $L^{*}$. Since 0 is a limit point of any such sequence and $f_{\alpha}\left[L_{\infty}\right]$ is closed, it follows that $0 \in f_{\alpha}\left[L_{\infty}\right]$. Therefore, some component of $L_{\infty}$ must be mapped into 0 . This contradicts the fact that $f_{\alpha}$ is an $\alpha$-mapping. Thus we may conclude that $q L^{*}<q L_{\infty}$.

Now suppose there is a set $X$ such that $q L^{*}<q X<q L_{\infty}$. Then $X$ has denumerably many components, $H_{1}, H_{2}, \cdots$, which are half-open intervals and no components which are open intervals, from Lemmas 2.3 and 2.8. moreover, every sequence of distinct $H_{i}$ 's contains a convergent subsequence (see [13, Theorem 7.1, p. 11]) which converges to some point of $X$ (otherwise $q L_{\infty} \leqq q X$ ). Furthermore, $S=\lim \sup \left\{H_{i} \mid i \in J\right\}$ is a totally disconnected subset of $X$ and we will show that $S$ is compact. Suppose $S$ is not compact. Then $S$ has a closed subset $Z$ which is homeomorphic to $J$. For each point $p_{j}$ of $Z$, we have that infinitely many $H_{i}$ 's are in each neighborhood of $p_{j}$. Let $N_{1}, N_{2}, \cdots$ be a collection of disjoint open intervals such that $\delta\left(N_{j}\right)<1 / j$ and $p_{j} \in N_{j}, j \in J$. Choose an $H_{i}$ in each $N_{j}$ and let $H$ be the union of these $H_{i}$. Then $H$ is a closed subset of $X$ which is homeomorphic to $L_{\infty}$. So $q L_{\infty} \leqq q H \leqq q X$ which is a contradiction. Therefore, $S$ is compact.

Let $\gamma$ be any covering of $X$. We proceed as in the proof of Theorem 2.10, except for taking $\Delta$ to be a denumerable dense subset of $R^{1}-X$ which contains the excluded end points of the half-open intervals which are components of $X$. With this modification we obtain as in the proof of Theorem 2.10:

(1) a covering $\beta$ of $X$ which refines $\gamma$, and

(2) a collection $V=\left\{K_{i}\right\}$ covering $X$ whose elements are open, closed, disjoint and $K_{i}=\left(a_{i}, b_{i}\right) \cap X$ where $a_{i}, b_{i} \in \Delta$.

Since $S$ is compact and the elements of $V$ are disjoint, finitely many elements of $V$ meet $S$, say $K_{1}, \cdots, K_{r}$. Let $K=\bigcup_{i=1}^{r} K_{i}$ and $Y=X-K$. Now $K$ contains all but a finite number of the components of $X$ which are half-open intervals. Therefore, $Y$ contains $m(<\infty)$ components of $X$ which are half-open intervals. Applying Theorem 2.26 we get that $q Y \leqq q L_{m+1}$ and, since $L_{m+1}$ is homeomorphic to $\Lambda_{m+1}=\bigcup_{i=1}^{m+1}\{[1 / 2 i, 1 / 2 i-1)\}, q Y \leqq q \Lambda_{m+1}$.

Now we will show that $q K \leqq q\left(L^{*}-\Lambda_{m+1}\right)$. For $i=1, \cdots, r$, each $K_{i}=\left(a_{i}, b_{i}\right) \cap X, a_{i}, b_{i} \in \Delta$, contains some $C_{j(i)}$, a half-open interval which is a component of $X$. Let $u_{i}=1 /(2 m+2 i+2)$ and $v_{i}=$ $1 /(2 m+2 i+1)$ for $i=1, \cdots, r$. Then we define a closed $\beta$-mapping $f_{\beta}$ of $K_{i}$ onto $\left[u_{i}, v_{i}\right)$ as follows: $\left(f_{\beta} \mid C_{j(i)}\right)$ is a homeomorphism of 
$C_{j(i)}$ onto $\left[u_{i}, v_{i}\right)$ and $f_{\beta}\left[K_{i}-C_{j(i)}\right]=u_{i}$, for $i=1, \cdots, r$. The proof that $f_{\beta}$ is a closed $\beta$-mapping is analogous to the proof given for the same purpose in Theorem 2.10. But $\beta>\gamma$ so $f_{\beta}$ is also a $\gamma$-mapping. Therefore, $q K \leqq q\left(L^{*}-\Lambda_{m+1}\right)$ and this together with the previously obtained relation, $q Y \leqq q \Lambda_{m+1}$, imply that $q X \leqq q L^{*}$ (by Theorem 1.2). This is a contradiction of our original assumption that $q L^{*}<q X<q L_{\infty}$, so we may conclude that $q L^{*} \ll q L_{\infty}$.

Theorem 2.28. $q X=q L^{*}$ if and only if (1) no component of $X$ is an open interval, (2) precisely denumerable many components of $X$ are half-open intervals, say $H_{1}, H_{2}, \ldots$ and (3) every sequence of distinct $H_{i}$ 's contains a subsequence which converges to some point of $X$.

Proof. Assume $q X=q L^{*}$. Then, by Lemma 2.3, $X$ does not have a component which is an open interval. By Lemma 2.8 (used twice), $X$ has precisely denumerably many components which are half-open intervals. Now suppose some sequence of distinct $H_{i}$ 's does not contain a subsequence which converges to a point of $X$. Then $X$ contains a closed subset homeomorphic to $L_{\infty}$. Therefore, $q L_{\infty} \leqq q X=$ $q L^{*}$ which contradicts Theorem 2.27. Hence every sequence of distinct $H_{i}$ 's contains a subsequence which converges to a point of $X$.

Now assume that conditions (1), (2) and (3) hold. Let $Y=H \cup\{p\}$ where $H$ is the union of a sequence of distinct $H_{i}$ 's which converges to $p$. Then $Y$ is homeomorphic to $L^{*}$. Hence $q L^{*} \leqq q X$.

Now let $\gamma$ be any covering of $X$. Since $S=\lim \sup \left\{H_{i} \mid i \in J\right\}$ is compact (by (3)), totally disconnected subset of $X$ we can proceed as in the latter part of the proof of Theorem 2.27 to obtain the relation $q X \leqq q L^{*}$. This together with the previously obtained relation $q L^{*} \leqq q X$ imply that $q X=q L^{*}$.

Theorem 2.29. $q X=q L_{\infty}$ if and only if (1) no component of $X$ is an open interval, (2) precisely denumerably many components of $X$ are half-open intervals, say $H_{1}, H_{2}, \cdots$, and (3) some sequence of distinct $H_{i}$ 's does not contain a subsequence which converges to a point of $X$.

Proof. Assume $q X=q L_{\infty}$. Then conditions (1) and (2) hold just as in the proof of Theorem 2.28. If condition (3) is not true, then by Theorem 2.28 and we have $q X=q L^{*}$. But this contradicts Theorem 2.27 and so condition (3) must hold.

Now assume that conditions (1), (2) and (3) hold. Condition (3) implies that $X$ contains a closed subset homeomorphic to $L_{\infty}$. Therefore, 
$q L_{\infty} \leqq q X$. Let $\gamma$ be any covering of $X$. We proceed as in the proof of Theorem 2.10, except for taking $\Delta$ to be a denumerable dense subset of $R^{1}-X$ which contains the excluded end points of the half-open intervals which are components of $X$. With this modification we obtain as in the proof of Theorem 2.10: (1) a covering $\beta$ of $X$ which refines $\gamma$, (2) a collection $V=\left\{K_{i}\right\}$ covering $X$ whose elements are open, closed, disjoint and $K_{i}=\left(a_{i}, b_{i}\right) \cap X$ where $a_{i}, b_{i} \in \Delta$.

We index the elements of $V$ as follows: even positive integers are used to index the totally disconnected elements of $V$ and odd positive integers are used to index the elements of $V$ which are not totally disconnected. We define a closed $\beta$-mapping, $f_{\beta}$, of $X$ into $L_{\infty}$ as follows:

(1) if $i$ is even, $f_{\beta}\left[K_{i}\right]=2 i$,

(2) if $i$ is odd and $K_{i}$ contains a half-open interval $C_{j}$, say $C_{j}=\left[c_{j}, d_{j}\right)$ which is a component of $X$,

$\left(f_{\beta} \mid C_{j}\right)$ is a homeomorphism of $C_{j}$ onto $(-2 i-1 / 2,-2 i]$

and $f_{\beta}\left[K_{i}-C_{j}\right]=-2 i$,

(3) if $i$ is odd and $K_{i}$ contains no such half-open interval, then $K_{i}$ contains a closed interval $C_{j}=\left[c_{j}, d_{j}\right]$, which is a component of $X$ and we define $\left(f_{\beta} \mid K_{i}\right)$ as an order preserving homeomorphism of $C_{j}$ onto

$$
\left.[-2 i-1 / 4,-2 i] ; f_{\beta}\left[\left(a_{i}, c_{j}\right) \cap X\right]=-2 i-1 / 4, f_{\beta}\left[d_{j}, b_{i}\right) \cap X\right]=-2 i .
$$

The proof that $f_{\beta}$ is a closed $\beta$-mapping is analogous to the proof given for the same purpose in Theorem 2.10. But $\beta>\gamma$ so $f_{\beta}$ is also $\gamma$-mapping of $X$ into $L_{\infty}$. Therefore, $q X \leqq q L_{\infty}$ and so $q X=q L_{\infty}$.

THEOREM 2.30. If $X$ does not have a component which is an open interval, then $q X \leqq q L_{\infty}$.

Proof. If $X$ has exactly $n(<\infty)$ components which are half-open intervals, then by Theorem 2.26 we have $q X \leqq q L_{n+1}$. If $X$ has precisely denumerably many components which are half-open intervals, then either Theorem 2.28 or Theorem 2.29 applies. Therefore, we have $q X \leqq q L^{*}$ or $q X \leqq q L_{\infty}$. But from any one of these three inequalities involving $q X$ it follows that $q X \leqq q L_{\infty}$.

DEFinition 2.2. Then $n$-chains $\mathscr{S}_{n}$. We call the following ordering of quasi dimension types the 0 -chain $\mathscr{S}_{0}$ (since there are no open intervals which are components of any of the sets in it). The sets inside the brackets are not comparable. (We call the spaces occuring here the standard 0-spaces.) 


$$
\begin{aligned}
\mathscr{S}_{0}: \quad q J_{0} & \ll q J_{1} \ll q J_{2} \ll \cdots \ll q J_{i} \ll q J_{i+1} \ll \cdots<q C \ll\left\{\begin{array}{l}
q I \\
q J
\end{array}\right\} \\
& \ll q(I \cup J) \ll q J^{*} \ll q L_{1} \ll q\left(L_{1} \cup J\right) \ll q\left(L_{1} \cup J^{*}\right) \ll q L_{2} \\
& \ll q\left(L_{1} \cup J\right) \ll q\left(L_{2} \cup J^{*}\right) \ll q L_{3} \ll \cdots \ll q L_{i} \ll q\left(L_{i} \cup J\right) \\
& \ll q\left(L_{i} \cup J^{*}\right) \ll q L_{i+1} \ll \cdots<q L^{*} \ll q L_{\infty} .
\end{aligned}
$$

The $n$-chain $\mathscr{S}_{n}$, for $n=1,2,3, \cdots$, is obtained from $\mathscr{S}_{0}$ by replacing each standard 0 -space by its union with $M_{n}$. (We call these the standard $n$-spaces.) So we have the following chain for $\mathscr{S}_{n}$.

$$
\begin{aligned}
\mathscr{S}_{n}: \quad q M_{n} & \ll q\left(M_{n} \cup J_{1}\right) \ll q\left(M_{n} \cup J_{2}\right) \ll \cdots \ll q\left(M_{n} \cup J_{i}\right) \\
& \ll q\left(M_{n} \cup J_{i+1}\right) \ll \cdots<q\left(M_{n} \cup C\right) \ll\left\{\begin{array}{l}
q\left(M_{n} \cup I\right) \\
q\left(M_{n} \cup J\right)
\end{array}\right\} \\
& \ll q\left(M_{n} \cup I \cup J\right) \ll q\left(M_{n} \cup J^{*}\right) \ll q\left(M_{n} \cup L_{1}\right) \\
& \ll q\left(M_{n} \cup L_{1} \cup J\right) \ll q\left(M_{n} \cup L_{1} \cup J^{*}\right) \ll q\left(M_{n} \cup L_{2}\right) \\
& \ll q\left(M_{n} \cup L_{2} \cup J\right) \ll q\left(M_{n} \cup L_{2} \cup J^{*}\right) \ll q\left(M_{n} \cup L_{3}\right) \\
& \ll \cdots \ll q\left(M_{n} \cup L_{i}\right) \ll q\left(M_{n} \cup L_{i} \cup J\right) \ll q\left(M_{n} \cup L_{i} \cup J^{*}\right) \\
& \ll q\left(M_{n} \cup L_{i+1}\right) \ll \cdots<q\left(M_{n} \cup L^{*}\right) \ll q\left(M_{n} \cup L_{\infty}\right) .
\end{aligned}
$$

Finally we define $\mathscr{S}_{\infty}: q M^{*} \ll q M_{\infty}$. (We call $M^{*}, M_{\infty}$ and the standard $n$-spaces, $n=0,1,2, \cdots$, the standard spaces.)

REMARK. Note that it follows from the previous theorems that every linear set with no components which are open intervals has the same quasi dimension type as some standard 0 -space.

THEOREM 2.3. The $n$-chains, for $n=0,1,2, \cdots$, have the ordering indicated in Definition 2.2 (where $q\left(M_{n} \cup C\right)$ and $q\left(M_{n} \cup L^{*}\right)$ are each the least upper bounds of their predecessors).

Proof. The previous results of $\S 2$ are proof of the case $n=0$. So we may consider $n>0$. Since $M_{n}$ is open and closed in its union with any standard 0 -space we can use Theorem 1.2 to get: if $X, Y$ are standard 0 -spaces and $q X<q Y$, then $q\left(M_{n} \cup X\right) \leqq q\left(M_{n} \cup Y\right)$. We wish to show that this last inequality is, in fact, a strict inequality. Suppose not, i.e., $q\left(M_{n} \cup Y\right) \leqq q\left(M_{n} \cup X\right)$. Then, for each covering $\alpha$ of $M_{n} \cup Y$, there is a closed $\alpha$-mapping, $f_{\alpha}$, of $M_{n} \cup Y$ into $M_{n} \cup X$. By the proof of Lemma 2.3 we have $f_{\alpha}\left[M_{n}\right] \supset M_{n}$, for $\alpha$ sufficiently fine. Therefore, $f_{\alpha}[Y] \subset X-M_{n}$, for $\alpha$ sufficiently fine, from which it follows that $q Y \leqq q\left(X-M_{n}\right)$. Since $X-M_{n}$ is a closed subset of $X$ we may conclude that $q Y \leqq q X$. This, however, is a contradiction and so we have $q\left(M_{n} \cup X\right)<q\left(M_{n} \cup Y\right)$. A similar argument shows that: if $X, Y$ are standard 0 -spaces which are not comparable, then 
$M_{n} \cup X$ and $M_{n} \cup Y$ are not comparable.

Now we wish to show the following: if $X, Y$ are standard 0 -spaces and $q X \ll q Y$, then $q\left(M_{n} \cup X\right) \ll q\left(M_{n} \cup Y\right)$. By the previous paragraph we need only show that there is no set $Z$ such that $q\left(M_{n} \cup X\right)<q Z<q\left(M_{n} \cup Y\right)$. Suppose not i.e., there is such a set $Z$. Then, by Lemma $2.3, Z$ contains exactly $n$ components $W_{1}, \cdots, W_{n}$ which are open intervals. Hence $Z=\bigcup_{i=1}^{n} W_{i} \cup Z^{\prime}$ where $q Z^{\prime}=q W$ for some standard 0 -space $T$ and so $q Z=q\left(M_{n} \cup T\right)$. Now since $q X \neq q T \neq q Y$ we have $q T<q X, q Y<q T$ or $T$ is not comparable to $X$ or $Y$ (but not both by the construction of $\mathscr{S}_{0}$ ). All there alternatives clearly lead to contradictions. Hence the $n$-chain $\mathscr{S}_{n}$ has the ordering indication in Definition 2.2.

THEOREM 2.32. $\mathscr{S}_{\infty}$ has the ordering indicated in Definition 2.2 (i.e., $q M^{*} \ll q M_{\infty}$ ).

Proof. Any covering $\alpha$ of $M^{*}$ can be refined by a covering $\beta=$ $\left\{B_{j} \mid j=0,1,2, \cdots\right\}$ such that:

(2) $B_{0}=[0,1 /(2 m+1)) \cap M^{*}$ for some $m \in J$,

(2) $B_{0} \cap B_{j}=\varnothing$ for $B_{j} \in \beta$ and $j \neq 0$.

Since $B=B_{0}$ is open and closed in $M^{*}$ so is $Y=\mathrm{U}_{j=1}^{\infty} B_{j}$. Moreover, $Y$ and $B$ are disjoint, $M^{*}=Y \cup B$ and

$$
Y=\bigcup\{(1 /(i+1), 1 / i) \mid i=1,3, \cdots, 2 m-1\} .
$$

We now define a closed $\beta$-mapping, $f_{\beta}$, of $M^{*}$ into $M_{2 m}$ (a closed subset of $\left.M_{\infty}\right)$. Let $f_{\beta}[B]=-4 m+3 / 4$ and $\left(f_{\beta} \mid(1 /(i+1), 1 / i)\right)$ be a homeomorphism of $(1 /(i+1,1 / i)$ onto $(-2 i+1 / 2,-2 i+1)$, for $i=$ $1,3, \cdots, 2 m-1$. Since $\beta>\alpha$ we have that $f_{\beta}$ is a closed $\alpha$-mapping of $M^{*}$ into $M_{\infty}$. Therefore, we may conclude that $q M^{*} \leqq q M_{\infty}$.

Now suppose that $q M_{\infty} \leqq q M^{*}$. Then, for any covering $\alpha$ of $M_{\infty}$, there is a closed $\alpha$-mapping, $f_{\alpha}$, of $M_{\infty}$ into $M^{*}$. Each component of $M_{\infty}$ con be written as the union of at least two distinct subsets which are open in $M_{\infty}$. Let $\alpha$ be any covering of $M_{\infty}$ of this type. Now $f_{\alpha}$ takes distinct components of $M_{\infty}$ into distinct components of $M^{*}$, for $\alpha$ sufficiently fine (this follows from the proof of Lemma 2.3). So $f_{\alpha}\left[M_{\infty}\right]$ must contain a sequence of distinct components of $M^{*}$. Since 0 is a limit point of any such sequence and $f_{\alpha}\left[M_{\infty}\right]$ is closed, it follows that $0 \in f_{\alpha}\left[M_{\infty}\right]$. Therefore, some component of $M_{\infty}$ must be mapped into 0 . This contradicts the fact that $f_{\alpha}$ is an $\alpha$-mapping. Thus we may conclude that $q M^{*}<q M_{\infty}$.

Now suppose there is a set $X$ such that $q M^{*}<q X<q M_{\infty}$. Then $X$ has denumerably many components, $W_{1}, W_{2}, \cdots$, which are open intervals. Moreover, every sequence of distinct $W_{i}$ 's contains a con- 
vergent subsequence which converges to some point of $X$ (otherwise $\left.q M_{\infty} \leqq q X\right)$. Furthermore, $S=\lim \sup \left\{W_{i} \mid i \in J\right\}$ is a totally disconnected subset of $X$ which is compact (see the proof of Theorem 2.27).

Let $\gamma$ be any covering of $X$. We proceed as in the proof of Theorem 2.10, except for taking $\Delta$ to be a denumerable dense subset of $R^{1}-X$ which contains the excluded end points of the open or halfopen intervals which are components of $X$. With this modification we obtain as in the proof of Theorem 2.10:

(1) a covering $\beta$ of $X$ which refines $\gamma$, and

(2) a collection $V=\left\{K_{i}\right\}$ covering $X$ whose elements are open, closed, disjoint and $K_{i}=\left(a_{i}, b_{i}\right) \cap X$ where $a_{i}, b_{i} \in \Delta$.

Since $S$ is compact and the elements of $V$ are disjoint, finitely many elements of $V$ meet $S$, say $K_{1}, \cdots, K_{r}$. Let $K=\bigcap_{i=1}^{r} K_{i}$ and $Y=X-K$. Now $K$ contains all but a finite number of the components of $X$ which are open intervals. So $Y$ has only $m(<\infty)$ components which are open intervals. Applying Theorem 2.31 we get that $q Y \leqq$ $q\left(M_{m} \cup L_{\infty}\right)$ and since

$$
q\left(M_{m} \cup L_{\infty}\right) \leqq q\left(M^{*}-\Omega_{r}\right)
$$

(where $\Omega_{r}=\bigcup_{i=1}^{r}\{(1 / 2 i, 1 / 2 i-1)\}$ ) we have $q Y \leqq q\left(M^{*}-\Omega_{r}\right)$.

Now we will show that $q K \leqq q \Omega_{r}$ by defining a closed $\beta$-mapping, $f_{\beta}$, of $K$ into $\Omega_{r}$ as follows:

(1) if $K_{i}$ contains a half-open interval $C_{j}$, say $C_{j}=\left[c_{j}, d_{j}\right)$, which is a component of $X$, then $\left(f_{\beta} \mid K_{i}\right)$ is a homeomorphism of $C_{j}$ onto $[2 /(4 i-1), 1 /(2 i-1))$ and $f_{\beta}\left[K_{i}-C_{j}\right]=2 /(4 i-1)$,

(2) if $K_{i}$ contains no such half-open interval, then $K_{i}$ contains a closed interval $C_{j}=\left[c_{j}, d_{j}\right]$ which is a component of $X$ and we define $f_{\beta}$ to be an order preserving homeomorphism of $C_{j}$ onto

$$
\begin{aligned}
& {[2 /(4 i-1), 4 /(8 i-1)] ; f_{\beta}\left[X \cap\left(a_{i}, c_{j}\right)\right]} \\
& \quad=2 /(4 i-1), f_{\beta}\left[X \cap\left(d_{j}, b_{i}\right)\right]=4 /(8 i-1) .
\end{aligned}
$$

The proof that $f_{\beta}$ is a closed $\beta$-mapping is analogous to the proof given for that purpose in Theorem 2.10. But $\beta>\gamma$ so $f_{\beta}$ is also a $\gamma$-mapping of $K$ into $\Omega_{r}$ and, therefore, $q K \leqq q \Omega_{r}$. This together with the relation $q Y \leqq q\left(M^{*}-\Omega_{r}\right)$ imply $q X \leqq q M^{*}$. However, this is a contradiction so we may conclude that $q M^{*} \ll q M_{\infty}$.

THeorem 2.33. $q X=q M^{*}$ if and only if (1) $X$ has precisely denumerably many components which are open intervals say $W_{1}$, $W_{2}, \cdots$ and (2) every sequence of distinct $W_{i}$ 's contains a subsequence which converges to some point of $X$.

Proof. First assume that $q X=q M^{*}$. Then Lemma 2.3 implies 
that $X$ has precisely denumerably many components which are open intervals, say $W_{1}, W_{2}, \cdots$. Now suppose some sequence of distinct $W_{i}$ 's does not contain a subsequence which converges to a point of $X$. Then $X$ contains a closed subset homeomorphic to $M_{\infty}$. Therefore, $q M_{\infty} \leqq q X=q M^{*}$ which contradicts the previous theorem. Hence (2) holds.

Now assume (1) and (2) hold. Let $Y=W \cup\{p\}$ where $W$ is the union of a sequence of distinct $W_{i}$ 's which converges to $p \in X$. Then $Y$ is homeomorphic to $M^{*}$. Hence $q M^{*} \leqq q X$.

Now let $\gamma$ be any covering of $X$. Since $S=\lim \sup \left\{W_{i} \mid i \in J\right\}$ is compact (by (2)), totally disconnected subset of $X$ we can proceed as in the latter part of the proof of Theorem 2.32 to obtain the relation, $q X \leqq q M^{*}$. This together with the previously obtained relation, $q M^{*} \leqq q X$ imply $q X=q M^{*}$.

THEOREM 2.34. $q X=q M_{\infty}$ if and only if (1) $X$ has precisely denumerably many components which are open intervals, say $W_{1}$, $W_{2}, \cdots$ and (2) some sequence of distinct $W_{i}$ 's does not contain a subsequence which converges to a point of $X$.

Proof. Assume $q X=q M_{\infty}$. The Lemma 2.3 implies that $X$ has precisely denumerably many components which are open intervals, say $W_{1}, W_{2}, \cdots$. Now suppose condition (2) does not hold. Then by the previous theorem we have $q X=q M^{*}$. But this contradicts Theorem 2.32 and so condition (2) must hold.

Now assume that conditions (1) and (2) hold. Condition (2) implies that $X$ contains a closed subset homeomorphic to $M_{\infty}$. Therefore, $q M_{\infty} \leqq q X$.

Now let $\gamma$ be any covering of $X$. We proceed as in the proof of Theorem 2.10, except for taking $\Delta$ to be a denumerable dense subset of $R^{1}-X$ which contains the excluded end points of the half-open or open intervals which are components of $X$. With this modification we obtain as in the proof of Theorem 2.10:

(1) a covering $\beta$ which refines $\gamma$,

(2) a collection $V=\left\{K_{i}\right\}$ covering $X$ whose elements are open, closed, disjoint and $K_{i}=\left(a_{i}, b_{i}\right) \cap X$ where $a_{i}, b_{i} \in \Delta$.

We index the elements $V$ as follows even positive integers are used to index the totally disconnected elements of $V$ and odd positive integers are used to index the elements of $V$ which are not totally disconnected. We now define a closed $\beta$-mapping, $f_{\beta}$, of $X$ into $M_{\infty}$ as follows:

(1) if $i$ is even, $f_{\beta}\left[K_{i}\right]=-2 i+3 / 4$,

(2) if $i$ is odd and $K_{i}$ contains a half-open interval $C_{j}$ (say 
$\left.C_{j}=\left[c_{j}, d_{j}\right)\right)$ which is a component of $K,\left(f_{\beta} \mid C_{j}\right)$ is a homeomorphism onto $[-2 i+3 / 4,-2 i+1)$ and $f_{\beta}\left[K_{i}-C_{j}\right]=-2 i+3 / 4$,

(3) if $i$ is odd and $K_{i}$ contains a closed interval $C_{j}=\left[c_{j}, d_{j}\right]$ which is a component of $X$, but no half-open interval which is a component of $X$, then we define $\left(f_{\beta} \mid K_{i}\right)$ as an order preserving homeomorphism of $C_{j}$ onto $[-2 i+3 / 8,-2 i+5 / 8] ; f_{\beta}\left[\left(a_{i}, c_{j}\right) \cap X\right]=$ $-2 i+3 / 8, f_{\beta}\left[\left(d_{j}, b_{i}\right) \cap X\right]=-2 i+5 / 8$,

(4) is $i$ is odd and $K_{i}$ contains an open interval $C_{j}=\left(c_{j}, d_{j}\right)$ which is a component of $X$, but no half-open or closed interval which is a component of $X$, then we define $\left(f_{\beta} \mid K_{i}\right)$ as an order preserving homeomorphism of $C_{j}$ onto $(-2 i+1 / 2,-2 i+1)$; if $c_{j} \neq a_{i}$, then $f_{\beta}\left[\left(a_{i}, c_{j}\right) \cap X\right]$ is a point of $f_{\beta}\left[B_{1} \cap C_{j}\right]$ where $B_{1}$ is a link of $\beta$ meeting $C_{j}$ with end point $a_{i}$; if $d_{j} \neq b_{i}$, then $f_{\beta}\left[\left(d_{j}, b_{i}\right) \cap X\right]$ is a point of $f_{\beta}\left[B_{2} \cap C_{j}\right]$ where $B_{2}$ is a link of $\beta$ meeting $C_{j}$ with end point $b_{i}$.

The proof that $f_{\beta}$ is a closed $\beta$-mapping is analogous to the proof given for the same purpose in Theorem 2.10. Since $\beta>\gamma$ we have that $f_{\beta}$ is also a $\gamma$-mapping of $X$ into $M_{\infty}$. Therefore, $q X \leqq q M_{\infty}$ and so $q X=q M_{\infty}$.

We next prove a theorem which determines all the order relationships between the $n$-chains. Together with the above theorem this determines completely the partial ordering of the quasi dimension types of subsets of $R^{1}$.

Lemma 2.9. If $q Y \leqq q X$ and $X$ has exactly $n$ components which are open intervals, then the number of components of $Y$ which are half-open intervals is at most $2 n$ more than the corresponding number for $X$. (This generalizes Lemma 2.8.)

Proof. First note that $q L_{3} \not \leq q M_{1}$ since, for any sufficiently fine cover $\alpha$ of $L_{3}$, the images of the components of $L_{3}$ under a closed $\alpha$-map would be mutually disjoint (each being a subinterval of $M_{1}$ of the form $(-3 / 2, y]$ or $[y,-1))$. Continuing in this manner we can show that $q L_{2 n+1} \not q M_{n}$ for any $n \in J$. Let $i$ be the number of components of $Y$ which are half-open intervals and let $k$ be the corresponding number for $X$.

Suppose the lemma is false, i.e., $i \geqq k+2 n+1$. Now for any covering $\alpha$ of $Y$ there is a closed $\alpha$-map $f_{\alpha}: Y \rightarrow X$. Then at most $2 n$ of the components of $Y$ which are half-open intervals map under $f_{\alpha}$ into $W$ (the union of the components of $X$ which are open intervals) for $\alpha$ sufficiently fine. So the other components of $Y$ which are halfopen intervals (of which there is at least $k+1$ ) map under $f_{\alpha}$ into $X-W$ for $\alpha$ sufficiently fine. It follows that $q L_{k+1} \leqq q(X-W) \leqq$ $q\left(L_{k} \cup J^{*}\right)$ which is a contradiction of Theorem 2.25. 
Lemma 2.10. If (1) $X$ and $M_{n}$ are separated, (2) $Y$ and $M_{n}$ and separated and (3) $q\left(M_{n} \cup Y\right) \leqq q\left(M_{n} \cup X\right)$, then $q Y \leqq q X$.

Proof. By (3) there is a closed $\alpha$-map $f_{\alpha}:\left(M_{n} \cup Y\right) \rightarrow\left(M_{n} \cup X\right)$ for every covering $\alpha$ of $M_{n} \cup Y$. As in the proof of Lemma 2.3 we have $f_{\alpha}\left[M_{n}\right] \supset M_{n}$, for $\alpha$ sufficiently fine. Therefore, $f_{\alpha}[Y] \subset X$, for $\alpha$ sufficiently fine. Since $X$ is a closed subset of $M_{n} \cup X$ and $Y$ is a closed subset of $M_{n} \cup Y$, it follows that $q Y \leqq q X$.

Lemma 2.11. The spaces $L_{\infty}$ and $M^{*}$ are not comparable.

Proof. By the proof of Lemma 2.3 we have that $q M^{*} \$ q L_{\infty}$. Suppose that $q L_{\infty} \leqq q M^{*}$. Then, for any covering $\alpha$ of $L_{\infty}$, there is a closed $\alpha$-mapping, $f_{\alpha}$, of $L_{\infty}$ into $M^{*}$. Now, since $q L_{3} \not \equiv q M_{1}$ (Lemma 2.9 ), at most two of the components of $L_{\infty}$ go into a single component of $M^{*}$ under $f_{\alpha}$, for $\alpha$ sufficiently fine. Therefore, $f_{\alpha}\left[L_{\infty}\right]$ contains denumerably many components of $M^{*}$ and so 0 is a limit point of $f_{\alpha}\left[L_{\infty}\right]$. Since $f_{\alpha}\left[L_{\infty}\right]$ is closed (in $M^{*}$ ) we have $0 \in f_{\alpha}\left[L_{\infty}\right]$. This implies that a component of $L_{\infty}$ is mapped into 0 which is a contraction of the fact that $f_{\alpha}$ is an $\alpha$-mapping (for $\alpha$ sufficiently fine). Therefore, we have $q L_{\infty} \not \leq q M^{*}$ and so $L_{\infty}$ and $M^{*}$ are not comparable.

THEOREM 2.35. Every linear set has the quasi dimension type of some (unique) standard space.

Proof. Suppose we are given a linear set $X$ and exactly $n(<\infty)$ of its components are open intervals $W_{1}, \cdots, W_{n}$ and $(<\infty)$ are halfopen intervals $H_{1}, \cdots, H_{m}$. Let $W=\bigcup_{i=1}^{n} W_{i}$ and $H=\bigcup_{i=1}^{m} H_{i}$ where if the upper limit is zero the union is understood to be the empty set. If $m \geqq 1$, then $Z=X-W$ is such that $q Z=q Y$ where

$$
Y=\left\{\begin{array}{c}
L_{m} \cup J^{*}, \text { if } \mathrm{Cl}(Z-H) \text { and } N(Z-H) \text { are not compact } \\
L_{m} \cup J, \text { if } \mathrm{Cl}(Z-H) \text { is not compact and } N(Z-H) \text { is } \\
\text { compact } \\
L_{m}, \text { if } \mathrm{Cl}(Z-H) \text { is compact }
\end{array}\right\} .
$$

If $m=0$, then $q Z=q Y$ where

$$
Y=\left\{\begin{array}{l}
J^{*}, \text { if } Z \text { and } N(Z) \text { are not compact } \\
I \cup J, \text { if } Z \text { is noncompact, not totally disconnected, } \\
\text { and } N(Z) \text { is compact } \\
J, \text { if } Z \text { is noncompact and totally disconnected } \\
I, \text { if } Z \text { is compact and not totally disconnected } \\
C, \text { if } Z \text { is compact, totally disconnected and infinite } \\
J_{i}, \text { if } Z \text { is finite and card } Z=i
\end{array}\right\}
$$


So $q X=q\left(M_{n} \cup Y\right)$ where $Y$ is given above.

Now we consider the cases when $m$ or $n$ is infinite. If $n<\infty$ and $m=\infty$ (i.e., $X$ has infinitely many components, $H_{1}, H_{2}, \cdots$, which are half-open intervals), then $q X=q\left(M_{n} \cup Y\right)$ where

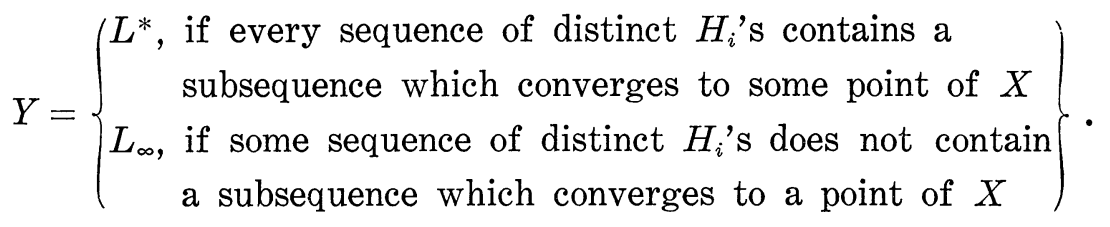

If $n=\infty$ (i.e., $X$ has infinitely many components, $W_{1}, W_{2}, \cdots$, which are open intervals), then

$$
q X=\left\{\begin{array}{c}
q M^{*}, \text { if every sequence of distinct } W_{i}^{\prime} \text { s contains a } \\
\text { subsequence which converges to some point of } X \\
q M_{\infty}, \text { if some sequence of distinct } W_{i}^{\prime} \text { s does not contain } \\
\text { a subsequence which converges to a point of } X
\end{array}\right\} .
$$

REMARK. It follows from Theorem 2.35 that for any linear set $X, q X \leqq q M_{\infty}$.

THEOREM 2.36. The only order relations between sets in different $\mathscr{S}_{n}$ 's are those implied by the following. For $i=2,3,4, \cdots$ and $j=[i / 2]$ (the largest integer $\leqq i / 2)$, we have

$$
\begin{aligned}
& \text { ( I ) } q L_{i} \ll q\left(M_{1} \cup L_{i-2}\right) \ll q\left(M_{2} \cup L_{i-4}\right) \ll \cdots \ll q\left(M_{j} \cup L_{i-2 j}\right) \text {, } \\
& \text { (II) } \quad q\left(L_{i} \cup J\right) \ll q\left(M_{1} \cup L_{i-2} \cup J\right) \ll q\left(M_{2} \cup L_{i-4} \cup J\right) \\
& \ll \cdots \ll q\left(M_{j} \cup L_{i-2 j} \cup J\right), \\
& \text { (III) } q\left(L_{i} \cup J^{*}\right) \ll q\left(M_{1} \cup L_{i-2} \cup J^{*}\right) \ll q\left(M_{2} \cup L_{i-4} \cup J^{*}\right) \\
& \ll \cdots \ll q\left(M_{j} \cup L_{i-2 j} \cup J^{*}\right), \\
& \text { (IV) } q L^{*} \ll q\left(M_{1} \cup L^{*}\right) \ll q\left(M_{2} \cup L^{*}\right) \ll \cdots \ll q\left(M_{n} \cup L^{*}\right) \\
& \ll q\left(M_{n+1} \cup L^{*}\right) \ll \cdots<q M^{*}, \\
& \text { ( V ) } q L_{\infty} \ll q\left(M_{1} \cup L_{\infty}\right) \ll q\left(M_{2} \cup L_{\infty}\right) \ll \cdots \ll q\left(M_{n} \cup L_{\infty}\right) \\
& \ll q\left(M_{n+1} \cup L_{\infty}\right) \ll \cdots<q M_{\infty} \text {. }
\end{aligned}
$$

Proof. In each case we have at least "ฏ”. Applying Lemma 2.3 we have " $<$ " in each case. Consider the first inequality in (I). Suppose there is a set $X$ such that $q L_{i}<q X<q\left(M_{1} \cup L_{i-2}\right)$. Then, by Lemma 2.3, $X$ has at most one component which is an open interval and so $q X \in \mathscr{S}_{1}$ or $q X \in \mathscr{S}_{0}$. The first alternative and Theorem 2.31 imply that $q X \leqq q\left(M_{1} \cup L_{i-3} \cup J^{*}\right)$. By assumption, $q L_{i}<q X$ so we have $q L_{i} \leqq q\left(M_{1} \cup L_{i-3} \cup J^{*}\right)$. But this contradicts Lemma 2.9. The second alternative and Theorem 2.31 imply that $q\left(L_{i} \cup J^{*}\right) \leqq q X$. By assumption $q X<q\left(M_{1} \cup L_{i-2}\right)$ so we have $q\left(L_{i} \cup J^{*}\right) \leqq q\left(M_{1} \cup L_{i-2}\right)$. 
Then, for any cover $\alpha$ of $L_{i} \cup J^{*}$, there is a closed $\alpha$-map

$$
f_{\alpha}:\left(L_{i} \cup J^{*}\right) \rightarrow\left(M_{1} \cup L_{i-2}\right)
$$

of $L_{i} \cup J^{*}$ into $M_{1} \cup L_{i-2}$. Since $q L_{i-1} \not \$ q L_{i-2}, f_{\alpha}^{-1}\left[M_{1}\right]$ contains at least two components of $L_{i}$ for $\alpha$ sufficiently fine. Since $q L_{3} \not \$ q M_{1}$, $f_{\alpha}^{-1}\left[M_{1}\right]$ contains at most two components of $L_{i}$ for $\alpha$ sufficiently fine. So, for $\alpha$ sufficiently fine, $f_{\alpha}^{-1}\left[M_{1}\right]$ contains exactly two components of $L_{i}$. It follows that $q\left(L_{i-2} \cup J^{*}\right) \leqq q L_{i-2}$ which contradicts Theorem 2.31. Hence $q L_{i} \ll q\left(M_{1} \cup L_{i-2}\right)$. The other inequalities of (I)-(III) can be obtained in a similar manner.

Consider the first inequality of (IV). Suppose there is a set $X$ such that $q L^{*}<q X<q\left(M_{1} \cup L^{*}\right)$. Now if $X$ does not contain a component which is an open interval, then by Theorem $2.30 q X \leqq q L_{\infty}$ and $q X=q L_{\infty}$. However, it follows from Lemma 2.11 that $q L_{\infty} \$$ $q\left(M_{1} \cup L^{*}\right)$ and so $X$ contains at least one component which is an open interval. Lemma 2.3 implies $X$ has exactly one such component. Since $q L^{*} \not \$ q\left(M_{1} \cup L_{n}\right)$ for any $n \in J$, we have that $X$ must contain denumerably many components which are half-open intervals. Hence, $q\left(M_{1} \cup L^{*}\right) \leqq q X$ which is a contradiction. Therefore, we may conclude that $q L^{*} \ll q\left(M_{1} \cup L^{*}\right)$. The other inequalities of (IV), except the last one, can be obtained in a similar manner. We now treat the last inequality of (IV). Suppose there is a set $X$ such that

$$
q\left(M_{n} \cup L^{*}\right)<q X<q M^{*}
$$

for all $n \in J$. Then $X$ must have denumerably many components which are open intervals by Lemma 2.3. Therefore, either Theorem 2.33 or Theorem 2.34 applies and so $q M^{*} \leqq q X$. This is a contradiction and so the last inequality of (IV) is valid.

Now consider the first inequality of (V). Suppose there is a set $X$ such that $q L_{\infty}<q X<q\left(M_{1} \cup L_{\infty}\right)$. Theorem 2.30 and Lemma 2.3 imply that $X$ has exactly one component, $W$, which is an open interval. Since $q L_{3} \not \$ W$, it follows that $q L_{\infty} \leqq q(X-W)$. The Theorem 1.2 applies and we have $q\left(M_{1} \cup L_{\infty}\right) \leqq q X$. This is a contradiction and so we may conclude that $q L_{\infty} \ll q\left(M_{1} \cup L_{\infty}\right)$. The other inequalities of (V), except the last one, can be obtained in a similar manner.

We now treat the last inequality of $(\mathrm{V})$. Suppose there is a set $X$ such that $q\left(M_{n} \cup L_{\infty}\right)<q X<q M_{\infty}$ for all $n \in J$. Then $X$ has denumerably many components which are open intervals. Therefore, $q X=q M^{*}$ or $q X=q M_{\infty}$. The latter is contrary to our assumption so we have $q X=q M^{*}$. However, it follows from Lemma 2.11 that $q\left(M_{n} \cup L_{\infty}\right) \not q M^{*}$, and so we have a contradiction. Thus we may conclude that the last inequality of $(\mathrm{V})$ is valid.

We now wish to show that: if $q Y<q X, q X \in \mathscr{S}_{n}$, and $q Y \in \mathscr{S}_{m}$, 
$0 \leqq m<n \leqq \infty$, then $q Y<q X$ can be obtained from (I)-(V), together with the orderings of the individual $\mathscr{S}_{i}$ 's. If $q Y<q\left(M_{m} \cup L_{1}\right)$, then using (I) we get

$$
\begin{aligned}
q Y<q\left(M_{m} \cup L_{2(n-m)}\right) & \ll q\left(M_{m+1} \cup L_{i(n-m)-2}\right) \\
& \ll q\left(M_{m+2} \cup L_{2(n-m)-4}\right) \ll \cdots \ll q M_{n} \leqq q X .
\end{aligned}
$$

Now assume that $q\left(M_{m} \cup L_{1}\right) \leqq q Y$. Then, either

(1) $q X=q\left(M_{n} \cup L_{k} \cup X_{1}\right)$ where $n, k<\infty$;

$$
X_{1}=J_{0}, J, \text { or } J^{*} \text {, }
$$

(2) $q X=q\left(M_{n} \cup L^{*}\right)$ where $n<\infty$,

(3) $q X=q\left(M_{n} \cup L_{\infty}\right)$ where $n<\infty$,

(4) $q X=q M^{*}$,

or

(5) $q X=q M_{\infty}$;

and either

(6) $q Y=q\left(M_{m} \cup L_{i} \cup Y_{1}\right)$ where $m<\infty, 1 \leqq i<\infty$;

$$
Y_{1}=J_{0}, J \text { or } J^{*} \text {, }
$$

(7) $q Y=q\left(M_{m} \cup L^{*}\right)$ where $m<\infty$,

(8) $q Y=q\left(M_{m} \cup L_{\infty}\right)$ where $m<\infty$,

or

(9) $q Y=q M^{*}$.

Since $q Y<q X$ none of the following pairs can hold together:

(1) $-(7),(1)-(8),(1)-(9)$,

( 2$)-(8),(2)-(9)$,

( 3$)-(9)$,

( 4$)-(8),(4)-(9)$.

The pair (5)-(9) does not apply since $q M^{*}$ and $q M_{\infty}$ are in the same chain: Let $r=i-2(n-m)$ and let $j=[i / 2]$. Then we proceed to consider the remaining cases.

Case 1. (1) and (6) hold. Then since $q Y<q X$, it follows from Lemmas 2.9 and 2.10 that $r \leqq k$. We have the following five subcases. Case 1.1. $Y_{1}=J_{0}$. If $r<0$, then $m+j<n$ so using (I) we have

$$
\begin{aligned}
q Y=q\left(M_{m} \cup L_{i}\right) & \ll q\left(M_{m+1} \cup L_{i-2}\right) \ll q\left(M_{m+2} \cup L_{i-4}\right) \\
& \ll \cdots \ll q\left(M_{m+j} \cup L_{i-2 j}\right) \leqq q\left(M_{m+j} \cup L_{1}\right) \\
& \leqq q M_{n} \leqq q X .
\end{aligned}
$$

On the other hand, if $r \geqq 0$, then using (I) we have

$$
\begin{aligned}
q Y=q\left(M_{m} \cup L_{i}\right) & \ll q\left(M_{m+1} \cup L_{i-}\right) \ll q\left(M_{m+2} \cup L_{i-4}\right) \\
& \ll \cdots \ll q\left(M_{n} \cup L_{r}\right) \leqq q\left(M_{n} \cup L_{k}\right) \leqq q X .
\end{aligned}
$$

Case 1.2. $Y_{1}=J$ and $X_{1}=J_{0}$. If $r<0$, then $m+j<n$ so using 
(II) and the fact that $q\left(L_{1} \cup J\right)<q M_{1}$ we have

$$
\begin{aligned}
q Y=q\left(M_{m} \cup L_{i} \cup J\right) \ll \cdots & \ll q\left(M_{m+j} \cup L_{i-2 j} \cup J\right) \\
& \leqq q\left(M_{m+j} \cup L_{1} \cup J\right) \leqq q M_{n} .
\end{aligned}
$$

On the otherhand, if $r>0$, then $r+1 \leqq k$. Otherwise, since $r \leqq k$ we would have $r=k$ and so $q\left(M_{m} \cup L_{i} \cup J\right)<q\left(M_{n} \cup L_{r}\right)$. This by Lemma 2.10 implies $q\left(L_{i} \cup J\right) \leqq q\left(M_{n-m} \cup L_{r}\right)$ from which it follows that $q L_{i+1} \leqq q\left(M_{n-m} \cup L_{r}\right)$. Applying Lemma 2.9 to the last relation we get $i+1 \leqq r+2(n-m)$, so that $i+1 \leqq i$. This is impossible so we have $r+1 \leqq k$. Then, using (II) we have

$$
\begin{aligned}
q Y=q\left(M_{m} \cup L_{i} \cup J\right) & \ll q\left(M_{m+1} \cup L_{i-2} \cup J\right) \\
& \ll \cdots \ll q\left(M_{n} \cup L_{r} \cup J\right) \\
& <q\left(M_{n} \cup L_{r+1}\right) \leqq q\left(M_{n} \cup L_{k}\right)=q X .
\end{aligned}
$$

Case 1.3. $Y_{1}=J$ and $X_{1}=J$ or $J^{*}$. If $r<0$ we can proceed just as in the first part of Case 1.2. If $r \geqq 0$, then using (II) and the fact that $r \leqq k$ we have

$$
\begin{aligned}
q Y=q\left(M_{m} \cup L_{i} \cup J\right) & \ll q\left(M_{m+1} \cup L_{i-2} \cup J\right) \\
& \ll \cdots \ll q\left(M_{n} \cup L_{r} \cup J\right) \\
& \leqq q\left(M_{n} \cup L_{k} \cup J\right) \leqq q X .
\end{aligned}
$$

Case 1.4. $Y_{1}=J^{*}$ and $X_{1}=J_{0}$ or $J$. This case is analogous to Case 1.2 except that (III) is used instead of (II).

Case 1.5. $Y_{1}=J^{*}$ and $X_{1}=J^{*}$. If $r<0$ we can proceed just as in the first part of Case 1.2. If $r \geqq 0$ then using (III) and the fact that $r \leqq k$ we have

$$
\begin{aligned}
q Y=q\left(M_{m} \cup L_{i} \cup J^{*}\right) & \ll q\left(M_{m+1} \cup L_{i-2} \cup J^{*}\right) \\
& \ll \cdots \ll q\left(M_{n} \cup L_{r} \cup J^{*}\right) \\
& \leqq q\left(M_{n} \cup L_{k} \cup J^{*}\right)=q X .
\end{aligned}
$$

Therefore, if (1) and (6) hold, we have that $q Y<q X$ is implied by (I)-(III).

Case 2. (2) and (6) hold. If $r<0$, then we can proceed just as in the first part of Case 1.2 except that (III) is used instead of (II). If $r \geqq 0$ then using (III) we have

$$
\begin{aligned}
q Y \leqq q\left(M_{m} \cup L_{i} \cup J^{*}\right) & \ll q\left(M_{m+1} \cup L_{i-2} \cup J^{*}\right) \ll \cdots \ll q\left(M_{n} \cup L_{r}\right) \\
& <q\left(M_{n} \cup L^{*}\right)=q X .
\end{aligned}
$$

Case 3. (2) and (7) hold. Then, using (IV) we have 


$$
q Y=q\left(M_{m} \cup L^{*}\right) \ll q\left(M_{m+1} \cup L^{*}\right) \ll \cdots \ll q\left(M_{n} \cup L^{*}\right)=q X .
$$

Case 4. (3) and (6) hold. If $r<0$, then we can proceed as in the first part of Case 1.2 except that (III) is used instead of (II). If $r \geqq 0$, then using (III) we have

$$
\begin{aligned}
q Y \leqq q\left(M_{m} \cup L_{i} \cup J^{*}\right) & \ll q\left(M_{m+1} \cup L_{i-2} \cup J^{*}\right) \ll \cdots \\
& \ll q\left(M_{n} \cup L_{r} \cup J^{*}\right)<q\left(M_{n} \cup L_{\infty}\right)=q X .
\end{aligned}
$$

Case 5. (3) and (7) hold. Then, using (IV) we have

$$
\begin{aligned}
q Y=q\left(M_{m} \cup L^{*}\right) & \ll q\left(M_{m+1} \cup L^{*}\right) \ll \cdots \\
& \ll q\left(M_{n} \cup L^{*}\right)<q\left(M_{n} \cup L_{\infty}\right)=q X .
\end{aligned}
$$

Case 6. (3) and (8) hold. Then, using (V) we have

$$
q Y=q\left(M_{m} \cup L_{\infty}\right) \ll q\left(M_{m+1} \cup L_{\infty}\right) \ll \cdots \ll q\left(M_{n} \cup L_{\infty}\right)=q X .
$$

Case 7. (4) and (6) hold. Proceed as in Case 4 and use (IV).

Case 8. (4) and (7) hold. Then, using (IV) we have

$$
q Y=q\left(M_{m} \cup L^{*}\right)<q M^{*}=q X .
$$

Case 9. (5) and (6) hold. Proceed as in Case 4 and use (V).

Case 10. (5) and (7) hold. Then, using (IV) we have

$$
q Y \leqq q\left(M_{m} \cup L^{*}\right)<q M^{*}<q M_{\infty}=q X .
$$

Case 11. (5) and (8) hold. Then, using (V) we have

$$
q Y=q\left(M_{m} \cup L_{\infty}\right)<q M_{\infty}=q X .
$$

Therefore, in all cases, $q Y<q X$ is implied by (I)-(V).

REMARK. We can characterize the class of linear sets with quasi dimension type $q\left(M_{n} \cup Y\right)$, for $n=0,1,2, \cdots$, where $Y$ is a standard 0 -space. For, if $X$ is a member of this class exactly $n$ of the components of $X$ are open intervals. Let $W$ denote the union of these $n$ components. Then, since $q(X-W)=q Y \in \mathscr{S}_{0}$ and we have already characterized such sets, we can obtain our desired topological characterization of linear sets of given quasi dimension type. Furthermore, if a linear set has quasi dimension type $q M^{*}$ or $q M_{\infty}$, then Theorem 2.33 and 2.34 give its topological characterization. 


\section{REFERENCES}

1. R. H. Bing, Snake-like continua, Duke Math J., 18 (1951), 653-663.

2. H. H. Corson, and J. R. Isbell, Some Properties of strong uniformities, Quar. J. Math. (2) 11 (1960).

3. M. Fréchet, Les dimensions d'un ensemble abstrait, Math. Ann. 68 (1910), 145-168.

4. - Les Espaces Abstraits, Gauthier-Villars, Paris, 1928.

5. H. Freudenthal, Entwicklungen von Räumen und ihre Gruppen, Comp. Math. 4 (1937), 145-234.

6. C. Kuratowski, Sur la puissance de l'ensemble des "nombres de dimension" au sens de M. Fréchet, Fund. Math. 8 (1926), 201-208.

7. C. Kuratowski and W. Sierpinski, Sur un problème de M. Fréchet concernant les dimensions des ensembles linéaires, Fund. Math. 8 (1926), 194-200.

8. C. Kuratowski and S. M. Ulam, Sur un coefficient lié aux transformations continues d'ensembles, Fund. Math. 20 (1933), 244-253.

9. C. N. Maxwell, An order relation among topological spaces, Trans. Amer. Math. Soc. 99 (1961), 201-204.

10. W. Sierpinski, Sur une propriété topologique des ensembles dénombrables denses en soi, Fund. Math. 1 (1920), 11-16.

11. - General Topology, University of Toronto Press, 1952.

12. K. Sitnikov, Example of a two-dimensional set in three-dimensional Euclidean space allowing arbitrarily small deformations into a 1-dimensional polyhedron and a certain new characteristic of the dimension of sets in Euclidean spaces, Doklady Akad. Nauk SSSR(N.S.) 88, 21-24 (1953) (Russian).

13. G. T. Whyburn, Analytic Topology, Amer. Math. Soc. Colloquium Publications, 1942.

Received August 20, 1963. The author was Supported in this research by the National Science Foundation NSFG-24421.

The UNIVERSity OF WASHINGTON

Seattle, Washington 


\title{
PACIFIC JOURNAL OF MATHEMATICS
}

\author{
EDITORS
}

H. Samelson

Stanford University

Stanford, California

J. P. JANS

University of Washington

Seattle, Washington 98105

\section{J. DugundJI}

University of Southern California Los Angeles, California 90007

RICHARD ARENS

University of California

Los Angeles, California 90024

\section{ASSOCIATE EDITORS}

E. F. BECKENBACH

B. H. NeUmanN

F. WOLF

K. YosIDA

\section{SUPPORTING INSTITUTIONS}

UNIVERSITY OF BRITISH COLUMBIA

CALIFORNIA INSTITUTE OF TECHNOLOGY

UNIVERSITY OF CALIFORNIA

MONTANA STATE UNIVERSITY

UNIVERSITY OF NEVADA

NEW MEXICO STATE UNIVERSITY

OREGON STATE UNIVERSITY

UNIVERSITY OF OREGON

OSAKA UNIVERSITY

UNIVERSITY OF SOUTHERN CALIFORNIA

\author{
STANFORD UNIVERSITY \\ UNIVERSITY OF TOKYO \\ UNIVERSITY OF UTAH \\ WASHINGTON STATE UNIVERSITY \\ UNIVERSITY OF WASHINGTON \\ * \\ AMERICAN MATHEMATICAL SOCIETY \\ CHEVRON RESEARCH CORPORATION \\ TRW SYSTEMS \\ NAVAL ORDNANCE TEST STATION
}

Mathematical papers intended for publication in the Pacific Journal of Mathematics should be typewritten (double spaced). The first paragraph or two must be capable of being used separately as a synopsis of the entire paper. It should not contain references to the bibliography. Manuscripts may be sent to any one of the four editors. All other communications to the editors should be addressed to the managing editor, Richard Arens at the University of California, Los Angeles, California 90024.

50 reprints per author of each article are furnished free of charge; additional copies may be obtained at cost in multiples of 50 .

The Pacific Journal of Mathematics is published monthly. Effective with Volume 16 the price per volume (3 numbers) is $\$ 8.00$; single issues, $\$ 3.00$. Special price for current issues to individual faculty members of supporting institutions and to individual members of the American Mathematical Society: $\$ 4.00$ per volume; single issues $\$ 1.50$. Back numbers are available.

Subscriptions, orders for back numbers, and changes of address should be sent to Pacific Journal of Mathematics, 103 Highland Boulevard, Berkeley 8, California.

Printed at Kokusai Bunken Insatsusha (International Academic Printing Co., Ltd.), No. 6, 2-chome, Fujimi-cho, Chiyoda-ku, Tokyo, Japan.

PUBLISHED BY PACIFIC JOURNAL OF MATHEMATICS, A NON-PROFIT CORPORATION

The Supporting Institutions listed above contribute to the cost of publication of this Journal, but they are not owners or publishers and have no responsibility for its content or policies. 


\section{Pacific Journal of Mathematics}

\section{Vol. 20, No. $3 \quad$ November, 1967}

Dallas O. Banks, Lower bounds for the eigenvalues of a vibrating string whose density satisfies a Lipschitz condition ................... 393

Ralph Joseph Bean, Decompositions of $E^{3}$ which yield $E^{3} \ldots \ldots \ldots \ldots 411$

Robert Bruce Brown, On generalized Cayley-Dickson algebras ......... 415

Richard Dowell Byrd, Complete distributivity in lattice-ordered groups . . . 423

Roger Countryman, On the characterization of compact Hausdorff $X$ for

which $C(X)$ is algebraically closed ...................... 433

Cecil Craig, Jr. and A. J. Macintyre, Inequalities for functions regular and bounded in a circle ...................................... 449

Takesi Isiwata, Mappings and spaces ..................... 455

David Lewis Outcalt, Power-associative algebras in which every subalgebra is an ideal ..................................... 481

Sidney Charles Port, Equilibrium systems of stable processes .......... 487

Jack Segal, Quasi dimension type. I. Types in the real line ............ 501

Robert William Stringall, Endomorphism rings of primary abelian

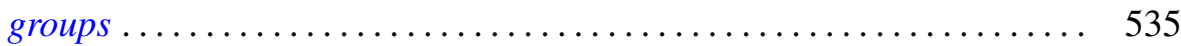

William John Sweeney, "The $\delta$-Poincaré estimate” ................. 559

L. Tzafriri, Operators commuting with Boolean algebras of projections of finite multiplicity ............................... 571 